Linearizations of Singular Matrix Polynomials and the Recovery of Minimal Indices

De Teran, Fernando and Dopico, Frolian M. and Mackey, D. Steven

2009

MIMS EPrint: 2009.25

Manchester Institute for Mathematical Sciences

School of Mathematics

The University of Manchester

\footnotetext{
Reports available from: http://eprints.maths.manchester.ac.uk/

And by contacting: The MIMS Secretary

School of Mathematics

The University of Manchester

Manchester, M13 9PL, UK
} 


\title{
LINEARIZATIONS OF SINGULAR MATRIX POLYNOMIALS AND THE RECOVERY OF MINIMAL INDICES *
}

\author{
FERNANDO DE TERÁN ${ }^{\dagger}$, FROILÁN M. DOPICO ${ }^{\ddagger}$, AND D. STEVEN MACKEY §
}

\begin{abstract}
A standard way of dealing with a regular matrix polynomial $P(\lambda)$ is to convert it into an equivalent matrix pencil - a process known as linearization. Two vector spaces of pencils $\mathbb{L}_{1}(P)$ and $\mathbb{L}_{2}(P)$ that generalize the first and second companion forms have recently been introduced by Mackey, Mackey, Mehl and Mehrmann. Almost all of these pencils are linearizations for $P(\lambda)$ when $P$ is regular. The goal of this work is to show that most of the pencils in $\mathbb{L}_{1}(P)$ and $\mathbb{L}_{2}(P)$ are still linearizations when $P(\lambda)$ is a singular square matrix polynomial, and that these linearizations can be used to obtain the complete eigenstructure of $P(\lambda)$, comprised not only of the finite and infinite eigenvalues, but also for singular polynomials of the left and right minimal indices and minimal bases. We show explicitly how to recover the minimal indices and bases of the polynomial $P(\lambda)$ from the minimal indices and bases of linearizations in $\mathbb{L}_{1}(P)$ and $\mathbb{L}_{2}(P)$. As a consequence of the recovery formulae for minimal indices, we prove that the vector space $\mathbb{D L}(P)=\mathbb{L}_{1}(P) \cap \mathbb{L}_{2}(P)$ will never contain any linearization for a square singular polynomial $P(\lambda)$. Finally, the results are extended to other linearizations of singular polynomials defined in terms of more general polynomial bases.
\end{abstract}

Key words. singular matrix polynomials, matrix pencils, minimal indices, minimal bases, linearization

AMS subject classifications. 15A18, 15A21, 15A22, 65F15

1. Introduction. Throughout this work we consider $n \times n$ matrix polynomials with degree $k$ of the form

$$
P(\lambda)=\sum_{i=0}^{k} \lambda^{i} A_{i}, \quad A_{0}, \ldots, A_{k} \in \mathbb{F}^{n \times n}, \quad A_{k} \neq 0,
$$

where $\mathbb{F}$ is the field of real or complex numbers. Our focus is on singular matrix polynomials. A matrix polynomial $P(\lambda)$ is said to be singular if $\operatorname{det} P(\lambda)$ is identically zero, and it is said to be regular otherwise. Square singular polynomials appear in practice, although not as frequently as regular polynomials. One well-known example is the study of differential-algebraic equations (see for instance [7] and the references therein). Other sources of problems involving singular matrix polynomials are control and linear systems theory [22], where the problem of computing minimal polynomial bases of null spaces of singular matrix polynomials is still the subject of intense research (see [3] and the references therein for an updated bibliography). In this context, it should be noted that the matrix polynomials arising in control are often fullrank rectangular polynomials. However square singular polynomials are also present in applications connected with linear systems [31].

${ }^{*}$ F. De Terán was partially supported by the Ministerio de Educación y Ciencia of Spain through grant MTM-2006-05361. F. M. Dopico was partially supported by the Ministerio de Educación y Ciencia of Spain through grant MTM-2006-06671 and the PRICIT program of Comunidad de Madrid through grant SIMUMAT (S-0505/ESP/0158). D. S. Mackey was partially supported by National Science Foundation grant DMS-0713799.

${ }^{\dagger}$ Departamento de Matemáticas, Universidad Carlos III de Madrid, Avda. Universidad 30, 28911 Leganés, Spain (fteran@math.uc3m.es).

${ }^{\ddagger}$ Instituto de Ciencias Matemáticas CSIC-UAM-UC3M-UCM and Departamento de Matemáticas, Universidad Carlos III de Madrid, Avda. Universidad 30, 28911 Leganés, Spain (dopico@math.uc3m.es).

$\S$ Department of Mathematics, Western Michigan University, Kalamazoo, MI 49008, USA (steve.mackey@wmich.edu). 
The standard way to numerically solve polynomial eigenvalue problems for regular polynomials $P(\lambda)$ is to first linearize $P(\lambda)$ into a matrix pencil $L(\lambda)=\lambda X+Y$ with $X, Y \in \mathbb{F}^{n k \times n k}$, and then compute the eigenvalues and eigenvectors of $L(\lambda)$. The classical approach is to use as linearizations the first and second companion forms [17]. However, companion forms may not share the structure of the original matrix polynomial. For instance, if the original polynomial is symmetric, skew-symmetric, or palindromic, the companion forms are not. Therefore the rounding errors inherent to numerical computations may destroy qualitative aspects of the spectrum. This has motivated the recent development of new classes of linearizations in [25] that have been further analyzed in [19, 20, 21, 26]. More recently, other classes of linearizations have been studied in $[1,2]$, some of which are closely connected to the ones in [25]. A different family of linearizations was introduced in $[4,5]$.

All of the new classes of linearizations introduced in $[2,4,25]$ have been studied only for regular matrix polynomials. It has been an open problem to determine if these pencils are linearizations when the square polynomial $P(\lambda)$ is singular, and, when they are, to investigate if they can be used to obtain the complete eigenstructure of $P(\lambda)$, i.e., the finite and infinite elementary divisors together with the left and right minimal indices $[14,22]$, and also to obtain the corresponding minimal bases. It is also an open problem to study if the new families of linearizations can be extended to rectangular matrix polynomials, a problem that may have relevant applications in structured problems in control and differential-algebraic equations. In this context it is interesting to note that minimal indices and bases arise in many problems in control $[14,22]$, and that their numerical computation is a hard problem that can be addressed in several different ways [3]. Among them, the companion linearization approach is one of the most reliable methods from a numerical point of view $[6,30]$.

In this work, we initiate the study of the extension of the new classes of linearizations to the case of square matrix polynomials that are singular. To keep the paper concise, we consider only the vector spaces of pencils introduced in [25] and those linearizations in [2] that are connected to the ones in [25]. Results concerning the family of linearizations introduced in [4] will be presented in the forthcoming paper [12]. In [25], two vector spaces of pencils $\mathbb{L}_{1}(P)$ and $\mathbb{L}_{2}(P)$ that generalize the first and second companion forms of the square polynomial $P(\lambda)$ were defined, and it was proved that almost all of the elements in these vector spaces are linearizations for $P(\lambda)$ when $P(\lambda)$ is regular. We will prove that this is also true when $P(\lambda)$ is a singular square matrix polynomial. In addition, we will show that the left and right minimal indices and bases of $P(\lambda)$ can be easily recovered from the minimal indices and bases of the linearizations in $\mathbb{L}_{1}(P)$ and $\mathbb{L}_{2}(P)$. All these results can be transferred to those pencils in [2, Sections 2 and 3] that are defined in terms of degree-graded polynomial bases, by showing that each of these pencils is strictly equivalent to some pencil in [25].

Finally, we will use the properties of the minimal index recovery formulae to prove that the vector space of pencils $\mathbb{D L}(P)=\mathbb{L}_{1}(P) \cap \mathbb{L}_{2}(P)$ does not contain any linearization of a square singular polynomial $P(\lambda)$. This result contrasts sharply with the situation for regular polynomials $P$, where almost every pencil in $\mathbb{D L}(P)$ is a (strong) linearization for $P$. The pencils in $\mathbb{D L}(P)$ also play a crucial role in the construction of all structure-preserving pencils in $\mathbb{L}_{1}(P)$ or $\mathbb{L}_{2}(P)$ for polynomials $P$ that are either symmetric, Hermitian, alternating, or palindromic [20, 26]. Consequently our results show that neither $\mathbb{L}_{1}(P)$ nor $\mathbb{L}_{2}(P)$ can ever provide any structured linearization for a singular polynomial $P$ with symmetric, Hermitian, alternating, or palindromic structure. 
The paper is organized as follows. In Section 2 we recall some basic concepts that are used throughout the paper. In addition, we show that strong linearizations of a singular matrix polynomial $P(\lambda)$ are the only matrix polynomials of degree one that allow even the possibility of recovering the complete eigenstructure of $P(\lambda)$. In Section 3 we establish relationships between the minimal indices and bases of a matrix polynomial and those of its reversal polynomial, and also between the left and right minimal indices and bases of symmetric, Hermitian, alternating, and palindromic polynomials; these relationships may be useful in other contexts. We prove in Section 4 that given a square singular matrix polynomial $P(\lambda)$, almost all pencils in the vector spaces $\mathbb{L}_{1}(P)$ and $\mathbb{L}_{2}(P)$ are strong linearizations for $P(\lambda)$, and then in Section 5 show how to recover the minimal indices and bases of $P(\lambda)$ from the corresponding quantities of these linearizations. These recovery results enable us to prove in Section 6 that none of the pencils in $\mathbb{D L}(P)$ is ever a linearization for a singular $P(\lambda)$. Recovery of minimal indices and bases of a singular polynomial from those of the linearizations introduced in [2, Sections 2 and 3] is then studied in Section 7; other related but more general linearizations are also considered. We close with some conclusions and discussion of future work in Section 8.

\section{Basic definitions and results.}

2.1. Null spaces, elementary divisors, and linearizations. We denote by $\mathbb{F}(\lambda)$ the field of rational functions with coefficients in $\mathbb{F}$, and by $\mathbb{F}(\lambda)^{n}$ the vector space of $n$-tuples with entries in $\mathbb{F}(\lambda)$. The normal rank of a matrix polynomial $P(\lambda)$, denoted nrank $P(\lambda)$, is the rank of $P(\lambda)$ considered as a matrix with entries in $\mathbb{F}(\lambda)$, or equivalently, the size of the largest non-identically zero minor of $P(\lambda)$ [15]. A finite eigenvalue of $P(\lambda)$ is a complex number $\lambda_{0}$ such that

$$
\text { rank } P\left(\lambda_{0}\right)<\operatorname{nrank} P(\lambda) \text {. }
$$

We say that $P(\lambda)$ has an infinite eigenvalue if the reversal polynomial

$$
\operatorname{rev} P(\lambda):=\lambda^{k} P(1 / \lambda)=\sum_{i=0}^{k} \lambda^{i} A_{k-i}
$$

has zero as eigenvalue.

An $n \times n$ singular matrix polynomial $P(\lambda)$ has right and left null vectors, that is, vectors $x(\lambda) \in \mathbb{F}(\lambda)^{n}$ and $y(\lambda) \in \mathbb{F}(\lambda)^{n}$ such that $P(\lambda) x(\lambda) \equiv 0$ and $y^{T}(\lambda) P(\lambda) \equiv 0$, where $y^{T}(\lambda)$ denotes the transpose of $y(\lambda)$. This leads to the following definition.

Definition 2.1. The right and left nullspaces of the $n \times n$ matrix polynomial $P(\lambda)$, denoted by $\mathcal{N}_{r}(P)$ and $\mathcal{N}_{\ell}(P)$ respectively, are the following subspaces of $\mathbb{F}(\lambda)^{n}$ :

$$
\begin{aligned}
& \mathcal{N}_{r}(P):=\left\{x(\lambda) \in \mathbb{F}(\lambda)^{n}: P(\lambda) x(\lambda) \equiv 0\right\}, \\
& \mathcal{N}_{\ell}(P):=\left\{y(\lambda) \in \mathbb{F}(\lambda)^{n}: y^{T}(\lambda) P(\lambda) \equiv 0\right\} .
\end{aligned}
$$

Note that we have the identity

$$
\operatorname{nrank}(P)=n-\operatorname{dim} \mathcal{N}_{r}(P)=n-\operatorname{dim} \mathcal{N}_{\ell}(P),
$$

and, in particular, $\operatorname{dim} \mathcal{N}_{r}(P)=\operatorname{dim} \mathcal{N}_{\ell}(P)$.

It is well known that the elementary divisors of $P(\lambda)$ (see definition in [15]) corresponding to its finite eigenvalues, as well as the dimensions of $\mathcal{N}_{r}(P)$ and $\mathcal{N}_{\ell}(P)$, are invariant under equivalence with respect to unimodular matrices, i.e., under preand post-multiplication by matrix polynomials with nonzero constant determinant 
[15]. The elementary divisors of $P(\lambda)$ corresponding to the infinite eigenvalue are defined as the elementary divisors corresponding to the zero eigenvalue of the reversal polynomial [18, Definition 1].

Next we recall the definition of linearization as introduced in [17], and also the related notion of strong linearization introduced in [16] and named in [23].

Definition 2.2. A matrix pencil $L(\lambda)=\lambda X+Y$ with $X, Y \in \mathbb{F}^{n k \times n k}$ is a linearization of an $n \times n$ matrix polynomial $P(\lambda)$ of degree $k$ if there exist two unimodular $n k \times n k$ matrices $E(\lambda)$ and $F(\lambda)$ such that

$$
E(\lambda) L(\lambda) F(\lambda)=\left[\begin{array}{cc}
P(\lambda) & 0 \\
0 & I_{(k-1) n}
\end{array}\right],
$$

or in other words, if $L(\lambda)$ is equivalent to $\operatorname{diag}\left(P(\lambda), I_{(k-1) n}\right)$. A linearization $L(\lambda)$ is called a strong linearization if $\operatorname{rev} L(\lambda)$ is also a linearization of rev $P(\lambda)$.

These definitions were introduced in $[16,17]$ only for regular polynomials; we are extending them here to square singular polynomials. In linear systems theory, some linearizations closely related to the companion forms have already been used for singular matrix polynomials [6, 30]. Recently [7], linearizations of possibly singular polynomials that may have smaller dimension than the ones in Definition 2.2 have been introduced in the context of differential algebraic equations. Following this idea, the minimal dimension for linearizations of a given matrix polynomial has been determined in [11].

Since Definition 2.2 was originally introduced only for regular matrix polynomials, one may wonder if linearizations are appropriate tools with which to study singular (square) matrix polynomials. The following result strongly indicates that the only $n k \times n k$ pencils from which one can reasonably hope to recover the complete eigenstructure of a singular polynomial $P$ are the strong linearizations of $P$.

Lemma 2.3. Let $P(\lambda)$ be an $n \times n$ matrix polynomial of degree $k$ and $L(\lambda)$ an $n k \times n k$ matrix pencil, and consider the following conditions on $L(\lambda)$ and $P(\lambda)$ :

(a) $\operatorname{dim} \mathcal{N}_{r}(L)=\operatorname{dim} \mathcal{N}_{r}(P)$,

(b) the finite elementary divisors of $L(\lambda)$ and $P(\lambda)$ are identical,

(c) the infinite elementary divisors of $L(\lambda)$ and $P(\lambda)$ are identical.

Then $L(\lambda)$ is

- a linearization of $P(\lambda)$ if and only if conditions (a) and (b) hold,

- a strong linearization of $P(\lambda)$ if and only if conditions (a), (b) and (c) hold.

Proof. Suppose first that $L(\lambda)$ is a linearization of $P(\lambda)$. Since nrank is preserved by unimodular transformations, it is immediate from (2.2) and Definition 2.2 that $\operatorname{dim} \mathcal{N}_{r}(L)=\operatorname{dim} \mathcal{N}_{r}(\operatorname{diag}(P, I))=\operatorname{dim} \mathcal{N}_{r}(P)$. Furthermore it is well known that elementary divisors associated to finite eigenvalues of a matrix polynomial are invariant under unimodular equivalence, so any linearization $L(\lambda)$ of a (regular or singular) matrix polynomial $P(\lambda)$ must have the same finite elementary divisors as $P(\lambda)$, but not necessarily the same infinite ones. When $L(\lambda)$ is a strong linearization, $\operatorname{rev} L(\lambda)$ and rev $P(\lambda)$ must also have the same finite elementary divisors, and so $L(\lambda)$ and $P(\lambda)$ have the same infinite elementary divisors. Thus we see that conditions (a) and (b) are necessary for an $n k \times n k$ pencil $L(\lambda)$ to be a linearization for $P(\lambda)$, while (a), (b) and (c) are necessary for $L(\lambda)$ to be a strong linearization.

Now suppose instead that $L(\lambda)$ is not a linearization of $P(\lambda)$. If the normal ranks of $L(\lambda)$ and $\operatorname{diag}(P(\lambda), I)$ differ, then $\operatorname{dim} \mathcal{N}_{r}(L) \neq \operatorname{dim} \mathcal{N}_{r}(P)$ immediately follows, and condition (a) is violated. If, on the other hand, $\operatorname{nrank} L(\lambda)=\operatorname{nrank} \operatorname{diag}(P(\lambda), I)$, then the number of invariant polynomials (see definition in [15]) of $L(\lambda)$ must be the 
same as the number of invariant polynomials of $\operatorname{diag}(P(\lambda), I)$, since for any matrix polynomial $Q$ this number is equal to $\operatorname{nrank} Q$. But the set of invariant polynomials of $\operatorname{diag}(P(\lambda), I)$ is just the invariant polynomials of $P(\lambda)$ together with $(k-1) n$ trivial invariant polynomials equal to one. By $[15$, Corollary 1, p. 141] two matrix polynomials are equivalent if and only if they have exactly the same invariant polynomials, so $L(\lambda)$ not being a linearization of $P(\lambda)$ means that the invariant polynomial sets of $L(\lambda)$ and $\operatorname{diag}(P(\lambda), I)$ must differ. Consequently the finite elementary divisors of $P(\lambda)$ and $L(\lambda)$ must also differ in some way, and so condition (b) is violated.

Finally suppose that $L(\lambda)$ is not a strong linearization of $P(\lambda)$. If this is because $L(\lambda)$ isn't even a linearization, then either condition (a) or (b) is violated. The only other possibility is that $L(\lambda)$ is a linearization of $P(\lambda)$ but not a strong linearization of $P(\lambda)$, i.e. $\operatorname{rev} L(\lambda)$ is not a linearization of $\operatorname{rev} P(\lambda)$. Since

$$
\operatorname{nrank}(\operatorname{rev} L(\lambda))=\operatorname{nrank} L(\lambda)=\operatorname{nrank} \operatorname{diag}(P(\lambda), I)=\operatorname{nrank} \operatorname{diag}(\operatorname{rev} P(\lambda), I),
$$

we have $\operatorname{dim} \mathcal{N}_{r}(\operatorname{rev} L)=\operatorname{dim} \mathcal{N}_{r}(\operatorname{diag}(\operatorname{rev} P, I))=\operatorname{dim} \mathcal{N}_{r}(\operatorname{rev} P)$, so condition (a) is satisfied for $\operatorname{rev} L(\lambda)$ and $\operatorname{rev} P(\lambda)$. But $\operatorname{rev} L(\lambda)$ is not a linearization of $\operatorname{rev} P(\lambda)$, so it must be that condition (b) is violated, and the finite elementary divisors of rev $L(\lambda)$ and $\operatorname{rev} P(\lambda)$ must differ in some way. However, it is not hard to see that if $L(\lambda)$ is a linearization of $P(\lambda)$ then the elementary divisors of $\operatorname{rev} L(\lambda)$ and $\operatorname{rev} P(\lambda)$ associated with any nonzero finite eigenvalue are the same. ${ }^{1}$ Thus the only possibility remaining is that the elementary divisors associated with the eigenvalue zero of $\operatorname{rev} L(\lambda)$ and rev $P(\lambda)$ are different. Hence the infinite elementary divisors of $L(\lambda)$ and $P(\lambda)$ are different, and condition (c) is violated.

Lemma 2.3 shows that strong linearizations of $P(\lambda)$ are the only matrix pencils of dimension $n k \times n k$ with both the same finite and infinite elementary divisors and the same null-space dimensions as $P(\lambda)$. Consequently for singular polynomials $P(\lambda)$, strong linearizations of $P(\lambda)$ seem to be the only good candidates among polynomials with degree one and dimension $n k \times n k$ from which to try to simultaneously recover all the spectral information of $P(\lambda)$, i.e., both the elementary divisors and the minimal indices. We will see in the remainder of this paper that this can in fact be achieved for at least some strong linearizations.

REMARK 1. In this paper we consider only the classical definition of linearization introduced in [17], i.e., linearizations with dimension $n k \times n k$. Recently [7], linearizations of dimension $s$ with $s \leq n k$ have been considered. The reader can easily check that the proof presented here for Lemma 2.3 remains valid for linearizations of dimension smaller than $n k$. The linearizations presented in [7] are not necessarily strong, indeed, they may have elementary divisors associated with the infinite eigenvalue of smaller degree than the corresponding ones in $P(\lambda)$. This means that the linearizations in [7] may be not useful for recovering the infinite elementary divisors of $P(\lambda)$, although they are still very interesting for other purposes.

2.2. Minimal indices and minimal bases. Eigenstructure of a singular polynomial. A vector polynomial is a vector whose entries are polynomials in the variable $\lambda$. For any subspace of $\mathbb{F}(\lambda)^{n}$, it is always possible to find a basis consisting entirely of vector polynomials; simply take an arbitrary basis and multiply each vector by the denominators of its entries. The degree of a vector polynomial is the greatest

\footnotetext{
${ }^{1}$ Recall that if $\lambda_{0} \neq 0$ is a finite eigenvalue of $P(\lambda)$ then the number and degrees of the elementary divisors of $P(\lambda)$ associated with $\lambda_{0}$ coincide with number and degrees of the elementary divisors of rev $P(\lambda)$ associated with $1 / \lambda_{0}$.
} 
degree of its components, and the order of a polynomial basis is defined as the sum of the degrees of its vectors [14, p. 494]. Then the following definition makes sense.

Definition 2.4. [14] Let $\mathcal{V}$ be a subspace of $\mathbb{F}(\lambda)^{n}$. A minimal basis of $\mathcal{V}$ is any polynomial basis of $\mathcal{V}$ with least order among all polynomial bases of $\mathcal{V}$.

It can be shown [14] that for any given subspace $\mathcal{V}$ of $\mathbb{F}(\lambda)^{n}$, the ordered list of degrees of the vector polynomials in any minimal basis of $\mathcal{V}$ is always the same. These degrees are then called the minimal indices of $\mathcal{V}$. Specializing $\mathcal{V}$ to be the left and right nullspaces of a singular matrix polynomial gives Definition 2.5; here $\operatorname{deg}(p(\lambda))$ denotes the degree of the vector polynomial $p(\lambda)$.

Definition 2.5. Let $P(\lambda)$ be a square singular matrix polynomial, and let the sets $\left\{y_{1}(\lambda), \ldots, y_{p}(\lambda)\right\}$ and $\left\{x_{1}(\lambda), \ldots, x_{p}(\lambda)\right\}$ be minimal bases of, respectively, the left and right nullspaces of $P(\lambda)$, ordered such that $\operatorname{deg}\left(y_{1}\right) \leq \operatorname{deg}\left(y_{2}\right) \leq \cdots \leq \operatorname{deg}\left(y_{p}\right)$ and $\operatorname{deg}\left(x_{1}\right) \leq \operatorname{deg}\left(x_{2}\right) \leq \cdots \leq \operatorname{deg}\left(x_{p}\right)$. Let $\eta_{i}=\operatorname{deg}\left(y_{i}\right)$ and $\varepsilon_{i}=\operatorname{deg}\left(x_{i}\right)$ for $i=1, \ldots, p$. Then $\eta_{1} \leq \eta_{2} \leq \cdots \leq \eta_{p}$ and $\varepsilon_{1} \leq \varepsilon_{2} \leq \cdots \leq \varepsilon_{p}$ are, respectively, the left and right minimal indices of $P(\lambda)$.

For the sake of brevity, we will call minimal bases of the left and right nullspaces of $P(\lambda)$ simply left and right minimal bases of $P(\lambda)$.

An alternative approach to defining the concepts of minimal indices and minimal bases of a subspace $\mathcal{V}$ of $\mathbb{F}(\lambda)^{n}$ can be found in [15, Chap. XII, Sec. 5], based on the following "greedy algorithm" for constructing a polynomial basis for $\mathcal{V}$. Begin by choosing any nonzero vector polynomial $z_{1}(\lambda)$ in $\mathcal{V}$ of minimal degree. Next choose any nonzero minimal degree vector polynomial $z_{2}(\lambda)$ in the complement $\mathcal{V} \backslash \operatorname{span}\left\{z_{1}(\lambda)\right\}$. Repeat the process with the complement $\mathcal{V} \backslash \operatorname{span}\left\{z_{1}(\lambda), z_{2}(\lambda)\right\}$, and so on, picking a nonzero minimal degree vector polynomial in the remaining complement at each step, until we get a basis of $\mathcal{V}$. The bases constructed in this way are clearly not unique, but the sequence of degrees of the vectors in any such basis is unique. Furthermore it can be shown [10, Lemma 2.4] that bases of $\mathcal{V}$ constructed in this way are in fact always minimal bases in the sense of Definition 2.4.

In the case of matrix pencils, the left (right) minimal indices coincide with the dimensions of the left (right) singular blocks of the Kronecker canonical form of the pencil [15, Chap. XII]. This canonical form can be stably computed through unitary transformations that lead to the GUPTRI form $[28,8,9,13]$. Therefore it is natural to look for relationships (if any) between the minimal indices of a singular matrix polynomial $P$ and the minimal indices of a given linearization, since this would provide a numerical method for computing the minimal indices of $P$. From the definition of linearization, one immediately sees that the number of left and right minimal indices of a matrix polynomial and the number of left and right minimal indices of any of its linearizations are the same. However, the values of these minimal indices may not coincide even for classical linearizations such as the companion forms. This is illustrated in the following example.

EXAMPLE 1 . Let $P(\lambda)$ be the $2 \times 2$ singular matrix polynomial of degree 2

$$
P(\lambda)=\left[\begin{array}{cc}
\lambda^{2} & \lambda \\
\lambda & 1
\end{array}\right]=\lambda^{2} A+\lambda B+C
$$

Then the first companion form of $P(\lambda)$, written $C_{1}(\lambda)=\lambda\left[\begin{array}{cc}A & 0 \\ 0 & I\end{array}\right]+\left[\begin{array}{cc}B & C \\ -I & 0\end{array}\right]$ 
as in $[25$, p. 974$]$, is

$$
C_{1}(\lambda)=\lambda\left[\begin{array}{llll}
1 & 0 & 0 & 0 \\
0 & 0 & 0 & 0 \\
0 & 0 & 1 & 0 \\
0 & 0 & 0 & 1
\end{array}\right]+\left[\begin{array}{rrrr}
0 & 1 & 0 & 0 \\
1 & 0 & 0 & 1 \\
-1 & 0 & 0 & 0 \\
0 & -1 & 0 & 0
\end{array}\right]=\left[\begin{array}{rrrr}
\lambda & 1 & 0 & 0 \\
1 & 0 & 0 & 1 \\
-1 & 0 & \lambda & 0 \\
0 & -1 & 0 & \lambda
\end{array}\right] .
$$

It is easy to see that $[1,-\lambda]^{T}$ is a minimal basis of $\mathcal{N}_{r}(P)$, so $P(\lambda)$ has exactly one right minimal index $\varepsilon_{1}=1$. By contrast $\left[\lambda,-\lambda^{2}, 1,-\lambda\right]^{T}$ is a minimal basis of $\mathcal{N}_{r}\left(C_{1}\right)$, so the single right minimal index of $C_{1}(\lambda)$ is $\varepsilon_{1}=2$.

Although the minimal indices of a matrix polynomial $P$ may differ from those of a given linearization, and in different ways for different linearizations, we will see that the minimal indices of $P$ are always related in a simple and uniform way to the minimal indices of any linearization in the families introduced in [25] and [2, Sections 2 and 3]. In addition, for these particular families of linearizations the minimal bases of the polynomial $P$ can always be easily recovered from the minimal bases of the linearizations. Recall that the linearizations in [25] include the companion forms.

In this paper we adopt the following definition.

Definition 2.6. The complete eigenstructure of a matrix polynomial $P(\lambda)$ consists of

1. its finite and infinite elementary divisors, and

2. its left and right minimal indices.

This definition extends to matrix polynomials the one introduced in [29] for pencils. It should be remarked that in linear systems theory other structural components related to the eigenvalues are also of interest [30].

Our overall goal, then, is given a singular matrix polynomial $P(\lambda)$, to find matrix pencils from which the complete eigenstructure of $P(\lambda)$ may be recovered. As Lemma 2.3 shows, it is natural to consider strong linearizations of $P(\lambda)$, because they allow us to obtain at least the elementary divisors and the nullspace dimensions. The remaining question for any given strong linearization is whether the minimal indices of $P(\lambda)$ may also be recovered.

3. Relationships among minimal indices. We start by establishing relationships between the minimal indices and bases of a matrix polynomial and those of its reversal polynomial. Although the reversal operation rev as defined in (2.1) is a straightforward notion, there are some simple but subtle aspects of this operation that should be kept firmly in mind. For example, rev is almost (but not quite) an involution. And viewed as a map on the space of all $m \times n$ matrix polynomials, rev is neither surjective nor injective. The extent to which these properties fail to hold is made precise in the next lemma, which is stated for general rectangular matrix polynomials so as to include the reversal of vector polynomials. We omit the proof, since all three parts are straightforward consequences of the definition of rev.

Lemma 3.1 (Properties of reversal). Let $P(\lambda)=\sum_{i=0}^{k} \lambda^{i} A_{i}$ be an $m \times n$ matrix polynomial of degree $k$, i.e., $A_{k}$ is the highest degree nonzero coefficient of $P(\lambda)$, and consider the operation rev defined by $\operatorname{rev} P(\lambda):=\lambda^{k} P(1 / \lambda)=\sum_{i=0}^{k} \lambda^{i} A_{k-i}$.

(a) If $k=\operatorname{deg} P(\lambda)$ and $\ell=\operatorname{deg}(\operatorname{rev} P(\lambda))$, then $\ell \leq k$, with equality if and only if $P(0) \neq 0_{m \times n}$. Furthermore $P(\lambda)=\lambda^{k-\ell} \cdot \operatorname{rev}(\operatorname{rev} P(\lambda))$.

(b) There exists an $m \times n$ matrix polynomial $Q(\lambda)$ such that $\operatorname{rev} Q(\lambda)=P(\lambda)$ if and only if $P(0) \neq 0_{m \times n}$. 
(c) Suppose $P(0) \neq 0_{m \times n}$. Then an $m \times n$ matrix polynomial $Q(\lambda)$ satisfies $\operatorname{rev} Q(\lambda)=P(\lambda)$ if and only if $Q(\lambda)=\lambda^{t} \cdot \operatorname{rev} P(\lambda)$ for some integer $t \geq 0$.

Observe that part (a) describes exactly the extent to which rev is (and isn't) an involution, part (b) characterizes the image of rev and its lack of surjectivity, while part (c) delineates the lack of injectivity of rev by characterizing the pre-image of any polynomial in the image of rev. In short, Lemma 3.1 shows that rev is an involutive bijection exactly if we restrict to the space of all $m \times n$ matrix polynomials $P(\lambda)$ such that $P(0) \neq 0_{m \times n}$. With these properties of rev in hand, we now develop the basic relationships between the minimal indices and bases of $P$ and those of rev $P$. In the remainder of this section we use the following notation: for a set of vector polynomials $\mathcal{B}=\left\{x_{1}(\lambda), \ldots, x_{p}(\lambda)\right\}$ in $\mathbb{F}(\lambda)^{n}$, the set $\left\{\operatorname{rev} x_{1}(\lambda), \ldots\right.$, rev $\left.x_{p}(\lambda)\right\}$ of the reversals of all the polynomials from $\mathcal{B}$ will be denoted by $\mathcal{B}_{\text {rev }}$.

THEOREM 3.2. Let $P(\lambda)$ be an $n \times n$ matrix polynomial of degree $k$, let $x(\lambda), y(\lambda)$, $z(\lambda), w(\lambda)$ be vector polynomials in $\mathbb{F}(\lambda)^{n}$, and let $\mathcal{B}$ and $\mathcal{E}$ be subsets of polynomials of $\mathbb{F}(\lambda)^{n}$. Then:

(a) $x(\lambda) \in \mathcal{N}_{r}(P) \Longleftrightarrow \operatorname{rev} x(\lambda) \in \mathcal{N}_{r}(\operatorname{rev} P)$, and $z(\lambda) \in \mathcal{N}_{r}(\operatorname{rev} P) \Longleftrightarrow \operatorname{rev} z(\lambda) \in \mathcal{N}_{r}(P)$.

(b) $y(\lambda) \in \mathcal{N}_{\ell}(P) \Longleftrightarrow \operatorname{rev} y(\lambda) \in \mathcal{N}_{\ell}(\operatorname{rev} P)$, and $w(\lambda) \in \mathcal{N}_{\ell}(\operatorname{rev} P) \Longleftrightarrow \operatorname{rev} w(\lambda) \in \mathcal{N}_{\ell}(P)$.

(c) $\mathcal{B}$ is a right (left) minimal basis of $P(\lambda) \Longrightarrow \mathcal{B}_{\text {rev }}$ is a right (left) minimal basis of $\operatorname{rev} P(\lambda)$, and the orders of the bases $\mathcal{B}$ and $\mathcal{B}_{\mathrm{rev}}$ are the same.

Also, $\mathcal{E}$ is a right (left) minimal basis of $\operatorname{rev} P(\lambda) \Longrightarrow \mathcal{E}_{\mathrm{rev}}$ is a right (left) minimal basis of $P(\lambda)$, and the orders of the bases $\mathcal{E}$ and $\mathcal{E}_{\text {rev }}$ are the same.

(d) The right (left) minimal indices of $P(\lambda)$ are equal to the right (left) minimal indices of $\operatorname{rev} P(\lambda)$.

Proof. We prove only the results pertaining to right null vectors, minimal indices and minimal bases; the arguments for left null vectors, minimal indices and minimal bases are similar. Begin by observing that even though the rev operation is not an involution, we have by Lemma 3.1(a) that $P(\lambda)=\lambda^{s} \cdot \operatorname{rev}(\operatorname{rev} P(\lambda))$ for some $s \geq 0$; as a consequence $\mathcal{N}_{r}(P)=\mathcal{N}_{r}(\operatorname{rev}(\operatorname{rev} P))$ always holds.

Now suppose that $x(\lambda) \in \mathcal{N}_{r}(P)$ has degree $q$. Then the equivalences

$$
\begin{aligned}
P(\lambda) x(\lambda)=0 \Longleftrightarrow P(1 / \lambda) x(1 / \lambda)=0 & \Longleftrightarrow \lambda^{k} P(1 / \lambda) \cdot \lambda^{q} x(1 / \lambda)=0 \\
& \Longleftrightarrow \operatorname{rev} P(\lambda) \cdot \operatorname{rev} x(\lambda)=0 .
\end{aligned}
$$

establish the first half of part (a). The second half of part (a) follows by applying the first half to the polynomial $Q(\lambda)=\operatorname{rev} P(\lambda)$.

$$
z(\lambda) \in \mathcal{N}_{r}(\operatorname{rev} P) \Longleftrightarrow \operatorname{rev} z(\lambda) \in \mathcal{N}_{r}(\operatorname{rev}(\operatorname{rev} P))=\mathcal{N}_{r}(P) .
$$

Next we turn to part (c), and start by showing that rev transforms any basis of $\mathcal{N}_{r}(P)$ into a basis of $\mathcal{N}_{r}($ rev $P)$, and vice versa. Consider first any set of vectors $\mathcal{B}=\left\{x_{1}(\lambda), \ldots, x_{p}(\lambda)\right\}$ in $\mathbb{F}(\lambda)^{n}$ with $\varepsilon_{i}=\operatorname{deg}\left(x_{i}(\lambda)\right)$, with the property that $\mathcal{B}_{\text {rev }}$ is linearly dependent. Hence there are rational functions $\alpha_{1}(\lambda), \ldots, \alpha_{p}(\lambda)$, not all zero, such that $\alpha_{1}(\lambda) \operatorname{rev} x_{1}(\lambda)+\cdots+\alpha_{p}(\lambda) \operatorname{rev} x_{p}(\lambda)=0$. Then

$$
\begin{aligned}
\sum_{i=1}^{p} \alpha_{i}(\lambda) \operatorname{rev} x_{i}(\lambda)=0 & \Longrightarrow \sum_{i=1}^{p} \alpha_{i}(\lambda) \lambda^{\varepsilon_{i}} x_{i}(1 / \lambda)=0 \\
& \Longrightarrow \sum_{i=1}^{p} \lambda^{-\varepsilon_{i}} \alpha_{i}(1 / \lambda) x_{i}(\lambda)=0 \Longrightarrow \sum_{i=1}^{p} \widetilde{\alpha}_{i}(\lambda) x_{i}(\lambda)=0
\end{aligned}
$$


with $\widetilde{\alpha}_{i}(\lambda):=\lambda^{-\varepsilon_{i}} \alpha_{i}(1 / \lambda)$ not all zero, showing that $\mathcal{B}=\left\{x_{1}(\lambda), \ldots, x_{p}(\lambda)\right\}$ must also be a linearly dependent set in $\mathbb{F}(\lambda)^{n}$. Thus we may conclude that rev preserves the property of linear independence; if $\mathcal{B}$ is any linearly independent set of vector polynomials, then $\mathcal{B}_{\text {rev }}$ is also linearly independent.

Next suppose that $\mathcal{B}=\left\{x_{1}(\lambda), \ldots, x_{p}(\lambda)\right\}$ with $\varepsilon_{i}=\operatorname{deg}\left(x_{i}(\lambda)\right)$ is a spanning set for $\mathcal{N}_{r}(P)$; we aim to show that $\mathcal{B}_{\text {rev }}$ is a spanning set for $\mathcal{N}_{r}($ rev $P)$. Letting $z(\lambda) \in$ $\mathcal{N}_{r}(\operatorname{rev} P)$ with $\operatorname{deg} z=\ell$ be arbitrary, then $\operatorname{rev} z(\lambda) \in \mathcal{N}_{r}(P)$ by part (a). Hence for some rational functions $\beta_{i}(\lambda)$ we have $\operatorname{rev} z(\lambda)=\beta_{1}(\lambda) x_{1}(\lambda)+\cdots+\beta_{p}(\lambda) x_{p}(\lambda)$. Then

$$
\begin{aligned}
\lambda^{\ell} z(1 / \lambda)=\sum_{i=1}^{p} \beta_{i}(\lambda) x_{i}(\lambda) & \Longrightarrow \lambda^{-\ell} z(\lambda)=\sum_{i=1}^{p} \beta_{i}(1 / \lambda) x_{i}(1 / \lambda) \\
& \Longrightarrow z(\lambda)=\sum_{i=1}^{p} \lambda^{\ell-\varepsilon_{i}} \beta_{i}(1 / \lambda) \cdot \lambda^{\varepsilon_{i}} x_{i}(1 / \lambda) \\
& \Longrightarrow z(\lambda)=\sum_{i=1}^{p} \widetilde{\beta}_{i}(\lambda) \operatorname{rev} x_{i}(\lambda)
\end{aligned}
$$

where $\widetilde{\beta}_{i}(\lambda):=\lambda^{\ell-\varepsilon_{i}} \beta_{i}(1 / \lambda)$, showing that $\mathcal{B}_{\text {rev }}$ spans $\mathcal{N}_{r}($ rev $P)$. Thus for any basis $\mathcal{B}$ of $\mathcal{N}_{r}(P)$, we see that $\mathcal{B}_{\text {rev }}$ will be a basis for $\mathcal{N}_{r}(\operatorname{rev} P)$.

Going in the other direction, let $\mathcal{E}=\left\{z_{1}(\lambda), \ldots, z_{p}(\lambda)\right\}$ be any basis for $\mathcal{N}_{r}(\operatorname{rev} P)$. Then by the above argument $\mathcal{E}_{\text {rev }}$ is a basis for $\mathcal{N}_{r}(\operatorname{rev}(\operatorname{rev} P))$, which was earlier shown to be the same as $\mathcal{N}_{r}(P)$.

Finally suppose that $\mathcal{B}=\left\{x_{1}(\lambda), \ldots, x_{p}(\lambda)\right\}$ is a minimal basis for $\mathcal{N}_{r}(P)$. Then each $x_{i}(\lambda)$ satisfies $x_{i}(0) \neq 0$, since otherwise $x_{i}(\lambda)=\lambda \cdot \widetilde{x}_{i}(\lambda)$ for some vector polynomial $\widetilde{x}_{i}(\lambda)$, and replacing $x_{i}(\lambda)$ by $\widetilde{x}_{i}(\lambda)$ would give a polynomial basis of lower order. Thus $\operatorname{deg}\left(\operatorname{rev} x_{i}(\lambda)\right)=\operatorname{deg} x_{i}(\lambda)$ for $i=1, \ldots, p$, and the order of the basis $\mathcal{B}_{\text {rev }}$ for $\mathcal{N}_{r}(\operatorname{rev} P)$ is the same as the order of $\mathcal{B}$. To see that $\mathcal{B}_{\text {rev }}$ is a minimal basis for $\mathcal{N}_{r}($ rev $P)$, suppose there was a basis $\mathcal{E}$ of $\mathcal{N}_{r}(\operatorname{rev} P)$ of lower order. Then $\mathcal{E}_{\text {rev }}$ would be a basis for $\mathcal{N}_{r}(P)$ of order strictly less than that of $\mathcal{B}$, contradicting the minimality of $\mathcal{B}$; this completes the proof of the first half of part (c). The second half of part (c) is established by a similar argument.

Part (d) now follows immediately from part (c) and the fact that $\operatorname{deg}\left(\operatorname{rev} x_{i}(\lambda)\right)=$ $\operatorname{deg} x_{i}(\lambda)$ for each vector polynomial $x_{i}(\lambda)$ in a minimal basis for $\mathcal{N}_{r}(P)$.

In the next definition we introduce several types of structured matrix polynomial that arise in a number of interesting applications [20, 26, 27]. For conciseness, the symbol $\star$ is used as an abbreviation for transpose $T$ in the real case and for either $T$ or conjugate transpose $*$ in the complex case.

DeFinition 3.3. Let $P(\lambda)$ be the $n \times n$ matrix polynomial as in (1.1), and define the associated polynomial $P^{\star}(\lambda)$ by

Then $P(\lambda)$ is said to be

$$
P^{\star}(\lambda):=\sum_{i=0}^{k} \lambda^{i} A_{i}^{\star} .
$$

- $\star$-symmetric if $P^{\star}(\lambda)=P(\lambda)$,

- $\star$-alternating if $P^{\star}(-\lambda)= \pm P(\lambda)$,

- $\star$-palindromic if $\operatorname{rev} P^{\star}(\lambda)=P(\lambda)$.

Theorems 3.4, 3.5, and 3.6 now establish relationships between the left and right minimal indices and bases of $\star$-symmetric, $\star$-alternating, and $\star$-palindromic matrix polynomials. Note in particular the structural feature common to all six types of matrix polynomial — the equality of left and right minimal indices. 
The following notation is used for these three theorems. If $x(\lambda)$ is a vector polynomial, let $\bar{x}(\lambda)$ denote the vector polynomial obtained from $x(\lambda)$ by complex conjugation of the coefficients. And for any set of vector polynomials $\mathcal{B}=\left\{x_{1}(\lambda), \ldots, x_{p}(\lambda)\right\}$ in $\mathbb{F}(\lambda)^{n}$, let $\overline{\mathcal{B}}, \mathcal{B}_{-}$, and $\mathcal{B}_{\text {rev }}$ denote the related sets $\overline{\mathcal{B}}:=\left\{\bar{x}_{1}(\lambda), \ldots, \bar{x}_{p}(\lambda)\right\}$, $\mathcal{B}_{-}:=\left\{x_{1}(-\lambda), \ldots, x_{p}(-\lambda)\right\}$, and $\mathcal{B}_{\text {rev }}:=\left\{\operatorname{rev} x_{1}(\lambda), \ldots, \operatorname{rev} x_{p}(\lambda)\right\}$.

THEOREM 3.4. Let $P(\lambda)$ be an $n \times n$ matrix polynomial, and $\mathcal{B}$ a set of vector polynomials in $\mathbb{F}(\lambda)^{n}$. If $P(\lambda)$ is T-symmetric, then

(a) $x(\lambda) \in \mathcal{N}_{r}(P) \Longleftrightarrow x(\lambda) \in \mathcal{N}_{\ell}(P)$,

(b) $\mathcal{B}$ is a minimal basis of $\mathcal{N}_{r}(P) \Longleftrightarrow \mathcal{B}$ is a minimal basis of $\mathcal{N}_{\ell}(P)$,

(c) the right minimal indices and left minimal indices of $P(\lambda)$ are the same.

If $P(\lambda)$ is *-symmetric, then

(d) $x(\lambda) \in \mathcal{N}_{r}(P) \Longleftrightarrow \bar{x}(\lambda) \in \mathcal{N}_{\ell}(P)$,

(e) $\mathcal{B}$ is a minimal basis of $\mathcal{N}_{r}(P) \Longleftrightarrow \overline{\mathcal{B}}$ is a minimal basis of $\mathcal{N}_{\ell}(P)$,

(f) the right minimal indices and left minimal indices of $P(\lambda)$ are the same.

Proof. When $P(\lambda)$ is $T$-symmetric, parts (a),(b), and (c) follow immediately from the equivalence $P(\lambda) x(\lambda)=0 \Leftrightarrow x^{T}(\lambda) P(\lambda)=0$. Parts (d), (e), and (f) follow from $P(\lambda) x(\lambda)=0 \Leftrightarrow \bar{x}^{T}(\lambda) P(\lambda)=0$, which holds when $P(\lambda)$ is $*$-symmetric.

Theorem 3.5. Let $P(\lambda)$ be an $n \times n$ matrix polynomial, and $\mathcal{B}$ a set of vector polynomials in $\mathbb{F}(\lambda)^{n}$. If $P(\lambda)$ is T-alternating, then

(a) $x(\lambda) \in \mathcal{N}_{r}(P) \Longleftrightarrow x(-\lambda) \in \mathcal{N}_{\ell}(P)$

(b) $\mathcal{B}$ is a minimal basis of $\mathcal{N}_{r}(P) \Longleftrightarrow \mathcal{B}_{-}$is a minimal basis of $\mathcal{N}_{\ell}(P)$,

(c) the right minimal indices and left minimal indices of $P(\lambda)$ are the same.

If $P(\lambda)$ is *-alternating, then

(d) $x(\lambda) \in \mathcal{N}_{r}(P) \Longleftrightarrow \bar{x}(-\lambda) \in \mathcal{N}_{\ell}(P)$

(e) $\mathcal{B}$ is a minimal basis of $\mathcal{N}_{r}(P) \Longleftrightarrow \overline{\mathcal{B}}_{-}$is a minimal basis of $\mathcal{N}_{\ell}(P)$,

(f) the right minimal indices and left minimal indices of $P(\lambda)$ are the same.

Proof. When $P(\lambda)$ is $T$-alternating, parts (a), (b), and (c) follow immediately from the equivalence $P(\lambda) x(\lambda)=0 \Leftrightarrow x^{T}(-\lambda) P^{T}(-\lambda)=0 \Leftrightarrow x^{T}(-\lambda) P(\lambda)=0$, together with the fact that $\operatorname{deg} x(\lambda)=\operatorname{deg} x(-\lambda)$. Parts (d), (e), and (f) follow from $P(\lambda) x(\lambda)=0 \Leftrightarrow x^{*}(-\lambda) P^{*}(-\lambda)=0 \Leftrightarrow \bar{x}^{T}(-\lambda) P(\lambda)=0$, which holds when $P(\lambda)$ is *-alternating, and $\operatorname{deg} x(\lambda)=\operatorname{deg} \bar{x}(-\lambda)$.

TheOREM 3.6. Let $P(\lambda)$ be an $n \times n$ matrix polynomial, and $\mathcal{B}$ a set of vector polynomials in $\mathbb{F}(\lambda)^{n}$. If $P(\lambda)$ is T-palindromic, then

(a) $x(\lambda) \in \mathcal{N}_{r}(P) \Longleftrightarrow \operatorname{rev} x(\lambda) \in \mathcal{N}_{\ell}(P), y(\lambda) \in \mathcal{N}_{\ell}(P) \Longleftrightarrow \operatorname{rev} y(\lambda) \in \mathcal{N}_{r}(P)$,

(b) $\mathcal{B}$ is a right (left) minimal basis of $P(\lambda)$

$\Longrightarrow \mathcal{B}_{\mathrm{rev}}$ is a left (right) minimal basis of $P(\lambda)$,

(c) the right minimal indices and left minimal indices of $P(\lambda)$ are the same.

If instead $P(\lambda)$ is *-palindromic, then results analogous to those above hold, but with rev $\bar{x}(\lambda)$ replacing rev $x(\lambda)$ and rev $\bar{y}(\lambda)$ replacing rev $y(\lambda)$ in part $(\mathrm{a})$, and $\overline{\mathcal{B}}_{\text {rev }}$ replacing $\mathcal{B}_{\text {rev }}$ in part (b).

Proof. Theorem 3.6 can be proved directly, but it can also be seen as a corollary of Theorem 3.2, because rev $P^{T}(\lambda)=P(\lambda)$ implies $\mathcal{N}_{r}(\operatorname{rev} P)=\mathcal{N}_{r}\left(P^{T}\right)=\mathcal{N}_{\ell}(P)$ and $\mathcal{N}_{\ell}(\operatorname{rev} P)=\mathcal{N}_{\ell}\left(P^{T}\right)=\mathcal{N}_{r}(P)$.

4. Linearizations for singular $P$ in the spaces $\mathbb{L}_{1}(P)$ and $\mathbb{L}_{2}(P)$. For the rest of the paper we follow the notation used in $[25]: \Lambda:=\left[\lambda^{k-1}, \lambda^{k-2}, \ldots, \lambda, 1\right]^{T}$ is the vector of decreasing powers of $\lambda$ and $\otimes$ denotes the Kronecker product. Now let $P(\lambda)$ be the matrix polynomial in (1.1), and consider the three vector spaces of 
$n k \times n k$ pencils $L(\lambda)=\lambda X+Y$ associated with $P$ that were introduced in [25]:

$$
\begin{aligned}
\mathbb{L}_{1}(P) & :=\left\{L(\lambda): L(\lambda)\left(\Lambda \otimes I_{n}\right)=v \otimes P(\lambda), v \in \mathbb{F}^{k}\right\}, \\
\mathbb{L}_{2}(P) & :=\left\{L(\lambda):\left(\Lambda^{T} \otimes I_{n}\right) L(\lambda)=w^{T} \otimes P(\lambda), w \in \mathbb{F}^{k}\right\}, \\
\mathbb{D L}(P) & :=\mathbb{L}_{1}(P) \cap \mathbb{L}_{2}(P) .
\end{aligned}
$$

The vectors $v$ and $w$ in (4.1) are referred to, respectively, as the "right ansatz" and "left ansatz" vectors of $L(\lambda)$. It is proved in [25] that, for any square matrix polynomial $P(\lambda)$, regular or singular, the sets in (4.1) are vector subspaces of the vector space of all $n k \times n k$ matrix pencils over $\mathbb{F}$. Letting $X_{1}=X_{2}=\operatorname{diag}\left(A_{k}, I_{n}, \ldots, I_{n}\right)$, and

$$
Y_{1}=\left[\begin{array}{cccc}
A_{k-1} & A_{k-2} & \cdots & A_{0} \\
-I_{n} & 0 & \cdots & 0 \\
& \ddots & \ddots & \vdots \\
0 & & -I_{n} & 0
\end{array}\right], \quad Y_{2}=\left[\begin{array}{cccc}
A_{k-1} & -I_{n} & & 0 \\
A_{k-2} & 0 & \ddots & \\
\vdots & \vdots & \ddots & -I_{n} \\
A_{0} & 0 & \cdots & 0
\end{array}\right] \text {, }
$$

then $C_{1}(\lambda):=\lambda X_{1}+Y_{1}$ and $C_{2}(\lambda):=\lambda X_{2}+Y_{2}$ are respectively the first and second companion forms of $P(\lambda)$. It is not hard to see that $C_{1}(\lambda) \in \mathbb{L}_{1}(P)$ with right ansatz vector $v=e_{1}$ (here $e_{1}$ denotes the first column of $I_{k}$ ), and $C_{2}(\lambda) \in \mathbb{L}_{2}(P)$ with left ansatz vector $w=e_{1}$. Indeed, the spaces $\mathbb{L}_{1}(P)$ and $\mathbb{L}_{2}(P)$ were specifically designed to be generalizations of $C_{1}(\lambda)$ and $C_{2}(\lambda)$.

In what follows we will see that the companion forms are far from being the only linearizations in $\mathbb{L}_{1}(P)$ and $\mathbb{L}_{2}(P)$, even in the case that $P(\lambda)$ is singular. It was shown in [25] that $\operatorname{dim} \mathbb{L}_{1}(P)=\operatorname{dim} \mathbb{L}_{2}(P)=k(k-1) n^{2}+k$, and we will prove in Theorem 4.4 that in fact almost all pencils in $\mathbb{L}_{1}(P)$ and $\mathbb{L}_{2}(P)$ are strong linearizations of $P$, regardless of whether $P(\lambda)$ is singular or not. The next result, which is a slight generalization of Theorem 4.1 in [25], gives us a simple way to detect strong linearizations in $\mathbb{L}_{1}(P)$.

THEOREM 4.1. Let $P(\lambda)=\sum_{i=0}^{k} \lambda^{i} A_{i}$ with $A_{k} \neq 0$ be a (regular or singular) $n \times n$ matrix polynomial and $L(\lambda)=\lambda X+Y \in \mathbb{L}_{1}(P)$ with right ansatz vector $v \neq 0$. Suppose $M \in \mathbb{F}^{k \times k}$ is any nonsingular matrix such that $M v=\alpha e_{1}$ for some number $\alpha \neq 0$. Then the pencil $\left(M \otimes I_{n}\right) L(\lambda)$ can be written as

$$
\left(M \otimes I_{n}\right) L(\lambda)=\lambda\left[\begin{array}{c|c}
\alpha A_{k} & X_{12} \\
\hline 0 & -Z
\end{array}\right]+\left[\begin{array}{c|c}
Y_{11} & \alpha A_{0} \\
\hline Z & 0
\end{array}\right],
$$

with $Z \in \mathbb{F}^{(k-1) n \times(k-1) n}$. If $Z$ is nonsingular then $L(\lambda)$ is a strong linearization of $P(\lambda)$.

Proof. Since $\left(M \otimes I_{n}\right)$ is a constant nonsingular matrix, clearly $L(\lambda)$ is a strong linearization of $P(\lambda)$ if and only if $\left(M \otimes I_{n}\right) L(\lambda)$ is too. Since $L(\lambda) \in \mathbb{L}_{1}(P)$ with right ansatz vector $v$, we have $L(\lambda)\left(\Lambda \otimes I_{n}\right)=v \otimes P(\lambda)$, so that multiplying by $M \otimes I_{n}$ yields

$$
\left(M \otimes I_{n}\right) L(\lambda)\left(\Lambda \otimes I_{n}\right)=M v \otimes P(\lambda)=\alpha e_{1} \otimes P(\lambda) .
$$

The result now follows by applying Theorem 4.1 in [25], valid for regular and singular $P(\lambda)$, to the matrix pencil $\left(M \otimes I_{n}\right) L(\lambda)$.

Theorem 4.1 shows that the nonsingularity of matrix $Z$ in (4.3) is a sufficient condition for $L(\lambda) \in \mathbb{L}_{1}(P)$ to be a strong linearization of $P(\lambda)$. In particular it shows 
that the first companion form $C_{1}(\lambda)$, with $Z=-I$, is always a strong linearization ${ }^{2}$, for regular or singular $P$. For regular polynomials this nonsingularity-of- $Z$ condition was also shown to be necessary in [25, Theorem 4.3], but this is no longer true when $P(\lambda)$ is singular, as the following example shows. This highlights an important difference between the cases of $P(\lambda)$ being regular or singular.

Example 2. Consider $P(\lambda)=\lambda^{2} A_{2}$ with $A_{2}=\left[\begin{array}{ll}1 & 0 \\ 0 & 0\end{array}\right]$, a singular matrix polynomial with one right minimal index $\varepsilon_{1}=0$ and one left minimal index $\eta_{1}=0$. Let $X_{12}=\left[\begin{array}{ll}0 & 0 \\ 0 & 1\end{array}\right]$ and $Z=\left[\begin{array}{cc}-1 & 0 \\ 0 & 0\end{array}\right]$. Then the pencil

$$
L(\lambda)=\lambda\left[\begin{array}{cc}
A_{2} & X_{12} \\
0 & -Z
\end{array}\right]+\left[\begin{array}{cc}
-X_{12} & 0 \\
Z & 0
\end{array}\right]
$$

is in $\mathbb{L}_{1}(P)$ with right ansatz vector $e_{1}$, which can be verified using either (4.1) or [25, Lemma 3.4]. Clearly $L(\lambda)$ has a singular $Z$ matrix, but it nevertheless is a strong linearization for $P(\lambda)$, because a single interchange of rows 2 and 4 turns $L(\lambda)$ into the first companion form $C_{1}(\lambda)$, which is always a strong linearization.

Given $L(\lambda) \in \mathbb{L}_{1}(P)$ with right ansatz vector $v \neq 0$, the matrix $M$ in the statement of Theorem 4.1 is not unique, so it is natural to ask whether different matrices $M_{1}$ and $M_{2}$ might lead to different matrices $Z_{1}$ and $Z_{2}$ in (4.3) with $Z_{1}$ being singular but $Z_{2}$ being nonsingular. It turns out that this is not possible. Indeed, the next result shows that for any given $L(\lambda) \in \mathbb{L}_{1}(P)$, all the matrices that can ever appear in the block labelled $Z$ in (4.3) have the same rank.

LEMMA 4.2. Let $P(\lambda)=\sum_{i=0}^{k} \lambda^{i} A_{i}$ with $A_{k} \neq 0$ be an $n \times n$ matrix polynomial and $L(\lambda)=\lambda X+Y \in \mathbb{L}_{1}(P)$ with right ansatz vector $v \neq 0$. Let $M_{1}, M_{2} \in \mathbb{F}^{k \times k}$ be two nonsingular matrices such that $M_{1} v=\alpha_{1} e_{1}$ and $M_{2} v=\alpha_{2} e_{1}$, for some numbers $\alpha_{1} \neq 0, \alpha_{2} \neq 0$. Let $Z_{1}, Z_{2} \in \mathbb{F}^{(k-1) n \times(k-1) n}$ be the corresponding matrices in the block labelled $Z$ in (4.3) associated with, respectively, $\left(M_{1}, \alpha_{1}\right)$ and $\left(M_{2}, \alpha_{2}\right)$. Then $\operatorname{rank} Z_{1}=\operatorname{rank} Z_{2}$.

Proof. Let $\left(\alpha_{1}, M_{1}, Z_{1}\right)$ and $\left(\alpha_{2}, M_{2}, Z_{2}\right)$ be two triples as in the statement of Theorem 4.1. The equality (4.3) can be applied to both triples, leading to two different expressions for $L(\lambda)$. Equating the coefficients of the first degree terms in these two expressions, we obtain

$$
\left(M_{1}^{-1} \otimes I_{n}\right)\left[\begin{array}{c|c}
\alpha_{1} A_{k} & X_{12} \\
\hline 0 & -Z_{1}
\end{array}\right]=\left(M_{2}^{-1} \otimes I_{n}\right)\left[\begin{array}{c|c}
\alpha_{2} A_{k} & \tilde{X}_{12} \\
\hline 0 & -Z_{2}
\end{array}\right],
$$

or equivalently

$$
\left[\begin{array}{c|c}
\alpha_{1} A_{k} & X_{12} \\
\hline 0 & -Z_{1}
\end{array}\right]=\left(M_{1} M_{2}^{-1} \otimes I_{n}\right)\left[\begin{array}{c|c}
\alpha_{2} A_{k} & \widetilde{X}_{12} \\
\hline 0 & -Z_{2}
\end{array}\right] .
$$

Note that $M_{1} v=\alpha_{1} e_{1}$ and $M_{2} v=\alpha_{2} e_{1}$ imply $\alpha_{1} M_{1}^{-1} e_{1}=\alpha_{2} M_{2}^{-1} e_{1}$, so $\left(\alpha_{1} / \alpha_{2}\right) e_{1}=$ $\left(M_{1} M_{2}^{-1}\right) e_{1}$. This means that the first column of $M_{1} M_{2}^{-1}$ is equal to $\left[\alpha_{1} / \alpha_{2}, 0, \ldots, 0\right]^{T}$. Then

$$
M_{1} M_{2}^{-1} \otimes I_{n}=\left[\begin{array}{c|c}
\frac{\alpha_{1}}{\alpha_{2}} I_{n} & Q_{12} \\
\hline 0 & Q_{22}
\end{array}\right]
$$

\footnotetext{
${ }^{2}$ That $C_{1}(\lambda)$ is always a strong linearization for regular $P$ was first shown in [16].
} 
where $Q_{22} \in \mathbb{F}^{(k-1) n \times(k-1) n}$ is nonsingular because $M_{1} M_{2}^{-1}$ is nonsingular. Now (4.4) implies $Z_{1}=Q_{22} Z_{2}$, so $\operatorname{rank}\left(Z_{1}\right)=\operatorname{rank}\left(Z_{2}\right)$ as claimed.

Lemma 4.2 shows that the rank of the block labelled $Z$ in (4.3) constitutes a well-defined property of pencils in $\mathbb{L}_{1}(P)$. Thus we introduce the following notions, that will be useful in Section 5 .

Definition 4.3. The $Z$-rank of $L(\lambda) \in \mathbb{L}_{1}(P)$ is the rank of any matrix appearing in the block labelled $Z$ in (4.3) under any reduction of $L(\lambda)$ of the form (4.3). If $Z$ in (4.3) is nonsingular, then we say that $L(\lambda) \in \mathbb{L}_{1}(P)$ has full $Z$-rank.

Theorem 4.1 shows that once a nonsingular matrix $M$ with $M v=\alpha e_{1}$ is fixed, a sufficient condition for a matrix pencil $L(\lambda) \in \mathbb{L}_{1}(P)$ with right ansatz vector $v$ to be a linearization of $P(\lambda)$ is that $Z$ in (4.3) is nonsingular. Since nonsingularity is a generic condition in matrix space, this suggests that most pencils in $\mathbb{L}_{1}(P)$ with right ansatz vector $v$ are linearizations of $P(\lambda)$, and, since $v$ is arbitrary, also that most pencils in $\mathbb{L}_{1}(P)$ are linearizations of $P(\lambda)$. It is known [25, Theorem 4.7] that this is true for regular $P(\lambda)$, that is: "almost every" pencil in $\mathbb{L}_{1}(P)$ is a linearization of $P(\lambda)$, where "almost every" means "for all but a closed, nowhere dense set of measure zero" in $\mathbb{L}_{1}(P)$. We will prove that this is also true for singular matrix polynomials. More precisely, we will exhibit a proper algebraic subset $\mathcal{A}$ of $\mathbb{L}_{1}(P)$ containing all the pencils in $\mathbb{L}_{1}(P)$ that are not linearizations of $P(\lambda)$, together with some linearizations. This will imply the result because proper algebraic sets are always closed, nowhere dense, and have measure zero. The subset $\mathcal{A}$ will be realized as the set of zeroes of a certain multivariate scalar-valued polynomial.

THEOREM 4.4 (Linearizations are generic in $\mathbb{L}_{1}(P)$ ).

For any $n \times n$ matrix polynomial $P(\lambda)$ of degree $k$ (regular or singular), almost every pencil in $\mathbb{L}_{1}(P)$ is a strong linearization for $P(\lambda)$.

Proof. Recall the following characterization of pencils in $\mathbb{L}_{1}(P)$ [25, Theorem 3.5]. Let $P(\lambda)=\sum_{i=0}^{k} \lambda^{i} A_{i}$ with $A_{k} \neq 0$ be an $n \times n$ matrix polynomial and $v \in \mathbb{F}^{k}$ any vector. Then the set of pencils in $\mathbb{L}_{1}(P)$ with right ansatz vector $v$ consists of all $L(\lambda)=\lambda X+Y$ such that

$$
\begin{aligned}
& X=\left[\begin{array}{cc}
n & (k-1) n \\
v \otimes A_{k} & -W
\end{array}\right] \\
& (k-1) n \quad n \\
& \text { and } \quad Y=\left[\begin{array}{lll}
\left.W+\left(\begin{array}{lll}
v \otimes[ & n \\
A_{k-1} & \ldots & A_{1}
\end{array}\right]\right) & v \otimes A_{0}
\end{array}\right]
\end{aligned}
$$

with $W \in \mathbb{F}^{k n \times(k-1) n}$ chosen arbitrarily.

This characterization allows us to parameterize $\mathbb{L}_{1}(P)$ by means of the isomorphism

$$
\begin{aligned}
\mathbb{L}_{1}(P) & \approx \mathbb{F}^{k} \times \mathbb{F}^{k n \times(k-1) n} \\
\lambda X+Y & \longmapsto
\end{aligned}
$$

Our goal is to find a single scalar-valued polynomial $\wp$ in the $k+k(k-1) n^{2}$ variables from $v$ and $W$ of $\mathbb{F}^{k} \times \mathbb{F}^{k n \times(k-1) n}$ such that all pencils in $\mathbb{L}_{1}(P)$ that are not linearizations are contained in the zero set $\mathcal{A}$ of $\wp$, and such that for some pencil in $\mathbb{L}_{1}(P)$ this polynomial is nonzero. Thus $\mathcal{A}$ will be a proper algebraic subset of $\mathbb{L}_{1}(P)$, and every pencil in the complement of $\mathcal{A}$ will be a strong linearization for $P(\lambda)$. The following construction gives a suitable polynomial $\wp$.

Suppose $L(\lambda)=\lambda X+Y$ is in $\mathbb{L}_{1}(P)$ with right ansatz vector $v=\left[v_{1}, \ldots, v_{k}\right]^{T}$. 
Let

$$
\widetilde{E}_{v}:=\left[\begin{array}{c|c}
1 & 0 \\
\hline-v_{2} & \\
\vdots & v_{1} I_{k-1} \\
-v_{k} &
\end{array}\right]
$$

and $E_{v}:=\widetilde{E}_{v} \otimes I_{n}$. Observe that $\widetilde{E}_{v} v=v_{1} e_{1}$, so if $v_{1} \neq 0$, then $\widetilde{E}_{v}$ is a particular selection of matrix $M$ in Theorem 4.1 with $\alpha=v_{1}$. Now $E_{v} L(\lambda)=E_{v}(\lambda X+Y)=$ $\lambda\left(E_{v} X\right)+\left(E_{v} Y\right)$, and from (4.5) we see that

$$
E_{v} X=\left[\widetilde{E}_{v} v \otimes A_{k} \mid-E_{v} W\right]=\left[\begin{array}{cc}
n & (k-1) n \\
v_{1} e_{1} \otimes A_{k} \mid & -E_{v} W
\end{array}\right] .
$$

Deleting the topmost $n$ rows of $-E_{v} W$ yields a $(k-1) n \times(k-1) n$ submatrix $B$ such that

$$
E_{v} X=\left[\begin{array}{c|c}
v_{1} A_{k} & * \\
\hline 0 & B
\end{array}\right]
$$

i.e., $-B$ is a $Z$-matrix as in (4.3). Now define $\wp:=v_{1} \cdot \operatorname{det} B$, which gives a polynomial in the $k+k(k-1) n^{2}$ variables defining $v$ and $W$; thus we may write $\wp(v, W)$. As a simple example note that the pair $(v, W)$ corresponding to the first companion form $C_{1}(\lambda) \in \mathbb{L}_{1}(P)$ has $v=e_{1}$ and $W=\left[0_{(k-1) n \times n} \mid-I_{(k-1) n}\right]^{T}$, and hence $B=I_{(k-1) n}$. Thus $\wp(v, W)=1 \neq 0$ for $C_{1}(\lambda)$, showing that the zero set of $\wp$ defines a proper algebraic subset $\mathcal{A}$ of $\mathbb{L}_{1}(P)$.

Clearly any pair $(v, W)$ such that $\wp(v, W) \neq 0$ has $v_{1} \neq 0$ and nonsingular $B$, so the corresponding $L(\lambda)$ will have full $Z$-rank and hence be a strong linearization by Theorem 4.1. Thus the complement of $\mathcal{A}$ contains only strong linearizations of $P$, and the proof is complete.

Corollary 4.5 follows immediately from the proof of Theorem 4.4. We will see in Section 5 that linearizations of full $Z$-rank are important in the recovery of minimal indices and bases. Therefore it is useful to know that full $Z$-rank pencils constitute almost all of $\mathbb{L}_{1}(P)$.

Corollary 4.5. For any (regular or singular) $n \times n$ matrix polynomial $P(\lambda)$ of degree $k$, almost every pencil in $\mathbb{L}_{1}(P)$ has full $Z$-rank.

It is worth noting that results analogous to Theorem 4.1, Lemma 4.2 and Theorem 4.4 also hold for matrix pencils in $\mathbb{L}_{2}(P)$. This is not surprising in light of the isomorphism of $\mathbb{L}_{1}(P)$ and $\mathbb{L}_{2}(P)$ via block transposition [20, Theorem 2.2]. We state without proof the analog of Theorem 4.1 for pencils in $\mathbb{L}_{2}(P)$ because this result will be used later. Note how block transposition establishes a simple correspondence between the statements of Theorem 4.1 and Theorem 4.6.

THEOREM 4.6. Let $P(\lambda)=\sum_{i=0}^{k} \lambda^{i} A_{i}$ with $A_{k} \neq 0$ be a (regular or singular) $n \times n$ matrix polynomial and $L(\lambda) \in \mathbb{L}_{2}(P)$ with left ansatz vector $w \neq 0$. Suppose $K \in \mathbb{F}^{k \times k}$ is any nonsingular matrix such that $w^{T} K=\alpha e_{1}^{T}$ for some number $\alpha \neq 0$. Then the pencil $L(\lambda)\left(K \otimes I_{n}\right)$ can be written as

$$
L(\lambda)\left(K \otimes I_{n}\right)=\lambda\left[\begin{array}{c|c}
\alpha A_{k} & 0 \\
\hline X_{21} & -Z
\end{array}\right]+\left[\begin{array}{c|c}
Y_{11} & Z \\
\hline \alpha A_{0} & 0
\end{array}\right]
$$

with $Z \in \mathbb{F}^{(k-1) n \times(k-1) n}$. If $Z$ is nonsingular then $L(\lambda)$ is a strong linearization of $P(\lambda)$. 
Theorem 4.6 together with the analog of Lemma 4.2 allows us to extend the notion of $Z$-rank to pencils in $\mathbb{L}_{2}(P)$ : the $Z$-rank of $L(\lambda) \in \mathbb{L}_{2}(P)$ is the rank of any matrix appearing in block $Z$ in (4.6) under any reduction of $L(\lambda)$ of the form (4.6).

We conclude this section with a brief discussion of linearizations versus strong linearizations in $\mathbb{L}_{1}(P)$. It was shown in $[25$, Theorem 4.3] that when $P(\lambda)$ is regular, any linearization in $\mathbb{L}_{1}(P)$ is necessarily a strong linearization. However, this is no longer true in the singular case. The following example provides a linearization for a singular polynomial $P(\lambda)$ that is in $\mathbb{L}_{1}(P)$, but is not a strong linearization.

EXAMPLE 3 . Let $P(\lambda)$ be the $2 \times 2$ quadratic matrix polynomial of Example 1 . Then the matrix pencil

$$
L(\lambda)=\left[\begin{array}{cccc}
\lambda & 1 & 0 & 0 \\
1 & 0 & 0 & 1 \\
1 & 0 & -\lambda & 0 \\
0 & 0 & 0 & 0
\end{array}\right]
$$

is in $\mathbb{L}_{1}(P)$ because $L(\lambda)\left(\Lambda \otimes I_{2}\right)=e_{1} \otimes P(\lambda)$, and it is also a linearization of $P(\lambda)$, because $E(\lambda) L(\lambda) F(\lambda)=\operatorname{diag}\left(P(\lambda), I_{2}\right)$ with

$$
E(\lambda)=\left[\begin{array}{cccc}
0 & 0 & \lambda & 1 \\
0 & 0 & 1 & 0 \\
1 & 0 & 0 & 0 \\
0 & 1 & 0 & 0
\end{array}\right] \text { and } F(\lambda)=\left[\begin{array}{cccc}
0 & 1 & 0 & 0 \\
0 & -\lambda & 1 & 0 \\
-1 & 0 & 0 & 0 \\
0 & -1 & 0 & 1
\end{array}\right]
$$

which are unimodular. On the other hand, the reversal pencil

$$
\operatorname{rev} L(\lambda)=\left[\begin{array}{cccc}
1 & \lambda & 0 & 0 \\
\lambda & 0 & 0 & \lambda \\
\lambda & 0 & -1 & 0 \\
0 & 0 & 0 & 0
\end{array}\right]
$$

has only one finite elementary divisor equal to $\lambda$, whereas the reversal polynomial $\operatorname{rev} P(\lambda)$ has no finite elementary divisors. Therefore by Lemma $2.3 \operatorname{rev} L(\lambda)$ cannot be a linearization for rev $P(\lambda)$, and so $L(\lambda)$ is not a strong linearization for $P(\lambda)$. As a consequence of Theorem 4.1 we know that $L(\lambda)$ cannot have full $Z$-rank; indeed, taking $M=I_{2}$ in (4.3) we have $Z=\left[\begin{array}{ll}1 & 0 \\ 0 & 0\end{array}\right]$, which is a singular matrix.

5. Recovery of minimal indices and bases from $\mathbb{L}_{1}(P)$ and $\mathbb{L}_{2}(P)$. As we have seen in Section 2 and Example 1, any linearization of an arbitrary (regular or singular) matrix polynomial $P(\lambda)$ will preserve the dimensions of the left and right nullspaces of $P(\lambda)$, that is, the number of left and right minimal indices, but not necessarily the values of these minimal indices. The main goal of this section is to analyze the relationship between the minimal indices and bases of $P(\lambda)$ and those of its linearizations in $\mathbb{L}_{1}(P)$ and $\mathbb{L}_{2}(P)$. We will prove that the right minimal indices of any linearization in $\mathbb{L}_{1}(P)$ (resp., the left minimal indices of any linearization in $\left.\mathbb{L}_{2}(P)\right)$ are all "shifted" from those of $P(\lambda)$ by $k-1$, where $k$ is the degree of the polynomial. We will also show that, for any linearization $L(\lambda)$ having full $Z$ rank, the left minimal indices of $L(\lambda) \in \mathbb{L}_{1}(P)$ (resp., the right minimal indices of $L(\lambda) \in \mathbb{L}_{2}(P)$ ) coincide exactly with those of $P(\lambda)$. These results will, in particular, allow us to recover the complete eigenstructure of any matrix polynomial from that of an arbitrary linearization of full $Z$-rank in $\mathbb{L}_{1}(P)$ or $\mathbb{L}_{2}(P)$. Finally we present some particular examples of linearizations in $\mathbb{L}_{1}(P)$ having defective $Z$-rank, but that 
still allow the recovery of the minimal indices of $P(\lambda)$. This shows that full $Z$-rank is a sufficient but not necessary condition for recovering the complete eigenstructure of a polynomial from linearizations in $\mathbb{L}_{1}(P)$ or $\mathbb{L}_{2}(P)$. All the results for recovering minimal indices are based on simple ways of recovering minimal bases of $P(\lambda)$ from those of its linearizations in $\mathbb{L}_{1}(P)$ and $\mathbb{L}_{2}(P)$.

5.1. Right minimal indices in $\mathbb{L}_{1}(P)$ and left minimal indices in $\mathbb{L}_{2}(P)$. We begin by establishing the basic relationship between right null vectors of $P(\lambda)$ and right null vectors of a linearization $L(\lambda) \in \mathbb{L}_{1}(P)$, extending the eigenvector recovery result for regular polynomials $P$ described in [25, Theorem 3.8] to singular polynomials $P$.

LEMma 5.1. Let $P(\lambda)$ be an $n \times n$ matrix polynomial of degree $k, L(\lambda) \in \mathbb{L}_{1}(P)$ with nonzero right ansatz vector $v$, and $x(\lambda) \in \mathbb{F}(\lambda)^{n}$. Then $\Lambda \otimes x(\lambda)$ is a right null vector of $L(\lambda)$ if and only if $x(\lambda)$ is a right null vector of $P(\lambda)$. Moreover, if $L(\lambda)$ is a linearization of $P(\lambda)$, then every right null vector of $L(\lambda)$ can be uniquely written as $\Lambda \otimes x(\lambda)$ for some right null vector $x(\lambda)$ of $P(\lambda)$, i.e., the mapping

$$
\begin{aligned}
\mathcal{R}_{\Lambda}: \mathcal{N}_{r}(P) & \longrightarrow \mathcal{N}_{r}(L) \\
x(\lambda) & \longmapsto \Lambda \otimes x(\lambda)
\end{aligned}
$$

is a linear isomorphism of $\mathbb{F}(\lambda)$-vector spaces. Furthermore, $\mathcal{R}_{\Lambda}$ induces a bijection between the vector polynomials in $\mathcal{N}_{r}(P)$ and the vector polynomials in $\mathcal{N}_{r}(L)$, i.e., $x(\lambda) \in \mathcal{N}_{r}(P)$ is a vector polynomial if and only if $\Lambda \otimes x(\lambda) \in \mathcal{N}_{r}(L)$ is a vector polynomial.

Proof. By the definition of $\mathbb{L}_{1}(P)$ in (4.1) we have $L(\lambda)\left(\Lambda \otimes I_{n}\right)=v \otimes P(\lambda)$; multiplying on the right by $x(\lambda)=1 \otimes x(\lambda)$ yields

$$
L(\lambda)(\Lambda \otimes x(\lambda))=v \otimes P(\lambda) x(\lambda)
$$

from which it then follows that $\Lambda \otimes x(\lambda) \in \mathcal{N}_{r}(L)$ if and only if $x(\lambda) \in \mathcal{N}_{r}(P)$. The map $\mathcal{R}_{\Lambda}$ is thus well-defined, and easily checked to be linear.

To prove the rest of the lemma, first recall that if $L(\lambda)$ is a linearization, then by Lemma 2.3 we have $\operatorname{dim} \mathcal{N}_{r}(P)=\operatorname{dim} \mathcal{N}_{r}(L)$. Thus $\mathcal{R}_{\Lambda}$ is an isomorphism if and only if $\operatorname{ker} \mathcal{R}_{\Lambda}=\{0\}$. So suppose $x(\lambda) \in \operatorname{ker} \mathcal{R}_{\Lambda}$, i.e. $0=\mathcal{R}_{\Lambda}(x(\lambda))=\Lambda \otimes x(\lambda)$. Clearly this implies $x(\lambda)=0$, since $x(\lambda)$ comprises the bottom $n$ entries of $\Lambda \otimes x(\lambda)$, and so $\operatorname{ker} \mathcal{R}_{\Lambda}=\{0\}$.

Finally, observe that the structure of $\Lambda$ guarantees that the $\mathbb{F}(\lambda)$-linear isomorphism $\mathcal{R}_{\Lambda}$ restricts to a bijection between the vector polynomials in $\mathcal{N}_{r}(P)$ and $\mathcal{N}_{r}(L)$; once again this follows from the fact that the bottom $n$ entries of $\Lambda \otimes x(\lambda)$ are just $x(\lambda)$ itself, together with the entries of $\Lambda$ being polynomials.

The definition of $\Lambda$ also implies that $\operatorname{deg}(\Lambda \otimes x(\lambda))=(k-1)+\operatorname{deg} x(\lambda)$ for any vector polynomial $x(\lambda)$, from which we obtain the following minimal index and minimal basis recovery result as an immediate consequence of Lemma 5.1.

TheOREm 5.2. Let $P(\lambda)$ be an $n \times n$ matrix polynomial of degree $k$, and let $L(\lambda) \in \mathbb{L}_{1}(P)$ be a linearization of $P(\lambda)$ with nonzero right ansatz vector.

(a) If $\varepsilon_{1} \leq \varepsilon_{2} \leq \cdots \leq \varepsilon_{p}$ are the right minimal indices of $P(\lambda)$, then

$$
(k-1)+\varepsilon_{1} \leq(k-1)+\varepsilon_{2} \leq \cdots \leq(k-1)+\varepsilon_{p}
$$

are the right minimal indices of $L(\lambda)$.

(b) Every minimal basis of $\mathcal{N}_{r}(L)$ is of the form $\left\{\Lambda \otimes x_{1}(\lambda), \ldots, \Lambda \otimes x_{p}(\lambda)\right\}$, where $\left\{x_{1}(\lambda), \ldots, x_{p}(\lambda)\right\}$ is a minimal basis of $\mathcal{N}_{r}(P)$. 
Clearly the structure of $\Lambda$ allows us to easily obtain a right minimal basis of $P(\lambda)$, simply by extracting the bottom $n$ entries from the $p$ vectors of any right minimal basis of $L(\lambda)$.

Analogous results for left minimal indices and bases of linearizations in $\mathbb{L}_{2}(P)$ are stated without proof, since the arguments proceed in a similar way.

Lemma 5.3. Let $P(\lambda)$ be an $n \times n$ matrix polynomial of degree $k, L(\lambda) \in \mathbb{L}_{2}(P)$ with nonzero left ansatz vector $w$, and $y(\lambda) \in \mathbb{F}(\lambda)^{n}$. Then $\Lambda \otimes y(\lambda)$ is a left null vector of $L(\lambda)$ if and only if $y(\lambda)$ is a left null vector of $P(\lambda)$. Moreover, if $L(\lambda)$ is a linearization of $P(\lambda)$, then every left null vector of $L(\lambda)$ can be uniquely written as $\Lambda \otimes y(\lambda)$ for some left null vector $y(\lambda)$ of $P(\lambda)$, i.e., the mapping

$$
\begin{aligned}
\mathcal{L}_{\Lambda}: \mathcal{N}_{\ell}(P) & \longrightarrow \mathcal{N}_{\ell}(L) \\
y(\lambda) & \longmapsto \Lambda \otimes y(\lambda)
\end{aligned}
$$

is a linear isomorphism of $\mathbb{F}(\lambda)$-vector spaces. Furthermore, $\mathcal{L}_{\Lambda}$ induces a bijection between the vector polynomials in $\mathcal{N}_{\ell}(P)$ and the vector polynomials in $\mathcal{N}_{\ell}(L)$, i.e., $y(\lambda) \in \mathcal{N}_{\ell}(P)$ is a vector polynomial if and only if $\Lambda \otimes y(\lambda) \in \mathcal{N}_{\ell}(L)$ is a vector polynomial.

THEOREM 5.4. Let $P(\lambda)$ be an $n \times n$ matrix polynomial of degree $k$, and let $L(\lambda) \in \mathbb{L}_{2}(P)$ be a linearization of $P(\lambda)$ with nonzero left ansatz vector.

(a) If $\eta_{1} \leq \eta_{2} \leq \cdots \leq \eta_{p}$ are the left minimal indices of $P(\lambda)$, then

$$
(k-1)+\eta_{1} \leq(k-1)+\eta_{2} \leq \cdots \leq(k-1)+\eta_{p}
$$

are the left minimal indices of $L(\lambda)$.

(b) Every minimal basis of $\mathcal{N}_{\ell}(L)$ is of the form $\left\{\Lambda \otimes y_{1}(\lambda), \ldots, \Lambda \otimes y_{p}(\lambda)\right\}$, where $\left\{y_{1}(\lambda), \ldots, y_{p}(\lambda)\right\}$ is a minimal basis of $\mathcal{N}_{\ell}(P)$.

The results in this section hold for any linearization $L(\lambda)$ in $\mathbb{L}_{1}(P)$ or in $\mathbb{L}_{2}(P)$, but they are incomplete because they do not provide any information on either the relationship between the left minimal indices and bases of $P$ and those of $L(\lambda) \in$ $\mathbb{L}_{1}(P)$, or the relationship between the right minimal indices and bases of $P$ and those of $L(\lambda) \in \mathbb{L}_{2}(P)$. These relationships are studied in the next section, but we will need to impose the mild condition of full $Z$-rank on the linearizations.

5.2. Left minimal indices in $\mathbb{L}_{1}(P)$ and right minimal indices in $\mathbb{L}_{2}(P)$. First we state two technical lemmas which are the analogs for left and right null vectors of Theorems 3.2 and 3.3 in [21].

Lemma 5.5. Let $P(\lambda)$ be an $n \times n$ matrix polynomial of degree $k$, and suppose $L(\lambda) \in \mathbb{L}_{1}(P)$ has full $Z$-rank and right ansatz vector $v \neq 0$. Then the mapping

$$
\begin{aligned}
\mathcal{L}_{v}: \mathcal{N}_{\ell}(L) & \longrightarrow \mathcal{N}_{\ell}(P) \\
y(\lambda) & \longmapsto\left(v^{T} \otimes I_{n}\right) y(\lambda)
\end{aligned}
$$

is a linear isomorphism of $\mathbb{F}(\lambda)$-vector spaces. Furthermore, $\mathcal{L}_{v}$ induces a bijection between the vector polynomials in $\mathcal{N}_{\ell}(L)$ and the vector polynomials in $\mathcal{N}_{\ell}(P)$, i.e., $y(\lambda) \in \mathcal{N}_{\ell}(L)$ is a vector polynomial if and only if $\left(v^{T} \otimes I_{n}\right) y(\lambda) \in \mathcal{N}_{\ell}(P)$ is a vector polynomial.

Proof. Since $L(\lambda)$ has right ansatz vector $v$ we have $L(\lambda)\left(\Lambda \otimes I_{n}\right)=v \otimes P(\lambda)$. Now if $y(\lambda) \in \mathcal{N}_{\ell}(L)$, then

$$
0=y^{T}(\lambda) L(\lambda)\left(\Lambda \otimes I_{n}\right)=y^{T}(\lambda)(v \otimes P(\lambda))=y^{T}(\lambda)\left(v \otimes I_{n}\right) P(\lambda),
$$


and so $\left(v^{T} \otimes I_{n}\right) y(\lambda) \in \mathcal{N}_{\ell}(P)$. Thus the mapping $\mathcal{L}_{v}$ is well defined, and easily checked to be a linear map between $\mathbb{F}(\lambda)$-vector spaces.

To verify the rest of the lemma, it is helpful to make use of the simplifying transformation provided by Theorem 4.1. Letting $M \in \mathbb{F}^{k \times k}$ be any nonsingular matrix such that $M v=e_{1}$, then by Theorem 4.1 we have

$$
\widehat{L}(\lambda):=\left(M \otimes I_{n}\right) L(\lambda)=\lambda\left[\begin{array}{c|c}
A_{k} & X_{12} \\
\hline 0 & -Z
\end{array}\right]+\left[\begin{array}{c|c}
Y_{11} & A_{0} \\
\hline Z & 0
\end{array}\right],
$$

where $Z \in \mathbb{F}^{(k-1) n \times(k-1) n}$ is nonsingular because $L(\lambda)$ is assumed to have full $Z$-rank. Now for $y(\lambda) \in \mathcal{N}_{\ell}(L)$, define

$$
z(\lambda):=\left(M^{-T} \otimes I_{n}\right) y(\lambda) .
$$

Then $z^{T}(\lambda) \widehat{L}(\lambda)=y^{T}(\lambda) L(\lambda)$, so $z(\lambda) \in \mathcal{N}_{\ell}(\widehat{L}) \Leftrightarrow y(\lambda) \in \mathcal{N}_{\ell}(L)$. Furthermore, if $z(\lambda)$ is partitioned as $\left[\begin{array}{ll}z_{1}^{T}(\lambda) & \widetilde{z}^{T}(\lambda)\end{array}\right]^{T}$ with $z_{1}(\lambda) \in \mathbb{F}(\lambda)^{n}$ and $\widetilde{z}(\lambda) \in \mathbb{F}(\lambda)^{(k-1) n}$, then

$$
\begin{aligned}
\mathcal{L}_{v}(y(\lambda))=\left(v^{T} \otimes I_{n}\right) y(\lambda) & =\left(v^{T} \otimes I_{n}\right)\left(M^{T} \otimes I_{n}\right) z(\lambda) \\
& =\left(e_{1}^{T} \otimes I_{n}\right) z(\lambda)=z_{1}(\lambda) .
\end{aligned}
$$

Thus if $y(\lambda) \in \mathcal{N}_{\ell}(L)$, then $z(\lambda) \in \mathcal{N}_{\ell}(\widehat{L})$, which can be rewritten as

$$
\left[\begin{array}{ll}
z_{1}^{T}(\lambda) & \widetilde{z}^{T}(\lambda)
\end{array}\right]\left(\lambda\left[\begin{array}{c|c}
A_{k} & X_{12} \\
\hline 0 & -Z
\end{array}\right]+\left[\begin{array}{c|c}
Y_{11} & A_{0} \\
\hline Z & 0
\end{array}\right]\right)=0 .
$$

Multiplying blockwise then yields

$$
z_{1}^{T}(\lambda)\left(\lambda\left[\begin{array}{ll}
A_{k} & X_{12}
\end{array}\right]+\left[\begin{array}{ll}
Y_{11} & A_{0}
\end{array}\right]\right)+\widetilde{z}^{T}(\lambda)\left(\lambda\left[\begin{array}{ll}
0 & -Z
\end{array}\right]+\left[\begin{array}{ll}
Z & 0
\end{array}\right]\right)=0,
$$

from which it follows that

$$
\widetilde{z}^{T}(\lambda)\left(\lambda\left[\begin{array}{ll}
0 & -Z
\end{array}\right]+\left[\begin{array}{ll}
Z & 0
\end{array}\right]\right)=-z_{1}^{T}(\lambda)\left(\lambda\left[\begin{array}{ll}
A_{k} & X_{12}
\end{array}\right]+\left[\begin{array}{ll}
Y_{11} & A_{0}
\end{array}\right]\right),
$$

or equivalently

$$
\widetilde{z}^{T}(\lambda) \cdot Z \cdot\left(S_{k}(\lambda) \otimes I_{n}\right)=-z_{1}^{T}(\lambda)\left(\lambda\left[\begin{array}{ll}
A_{k} & X_{12}
\end{array}\right]+\left[\begin{array}{ll}
Y_{11} & A_{0}
\end{array}\right]\right),
$$

where $S_{k}(\lambda)$ is the rectangular $(k-1) \times k$ pencil

$$
S_{k}(\lambda)=\lambda\left[\begin{array}{ll}
0 & -I_{k-1}
\end{array}\right]+\left[\begin{array}{ll}
I_{k-1} & 0
\end{array}\right]=\left[\begin{array}{ccccc}
1 & -\lambda & & & \\
& 1 & -\lambda & & \\
& & \ddots & \ddots & \\
& & & 1 & -\lambda
\end{array}\right] .
$$

But $S_{k}(\lambda)$ has a polynomial $k \times(k-1)$ Toeplitz right inverse given by

$$
S_{k}^{\sharp}(\lambda)=\left[\begin{array}{ccccc}
1 & \lambda & \lambda^{2} & \cdots & \lambda^{k-2} \\
0 & 1 & \lambda & \ddots & \vdots \\
& & \ddots & \ddots & \lambda^{2} \\
\vdots & & & \ddots & \lambda \\
& & & & 1 \\
0 & & \cdots & & 0
\end{array}\right],
$$


so that $S_{k}(\lambda) S_{k}^{\sharp}(\lambda)=I_{k-1}$. Hence we can solve (5.6) for $\widetilde{z}^{T}(\lambda)$ to get

$$
\widetilde{z}^{T}(\lambda)=-z_{1}^{T}(\lambda)\left(\lambda\left[\begin{array}{ll}
A_{k} & X_{12}
\end{array}\right]+\left[\begin{array}{ll}
Y_{11} & A_{0}
\end{array}\right]\right) \cdot\left(S_{k}^{\sharp}(\lambda) \otimes I_{n}\right) \cdot Z^{-1} .
$$

From (5.7) the rest of the lemma now quickly follows. Since $L(\lambda)$ has full $Z$ rank, Theorem 4.1 guarantees that $L(\lambda)$ is a strong linearization of $P(\lambda)$, and so $\operatorname{dim} \mathcal{N}_{\ell}(L)=\operatorname{dim} \mathcal{N}_{\ell}(P)$. Thus to conclude that $\mathcal{L}_{v}$ is an isomorphism, it suffices to show that $\operatorname{ker} \mathcal{L}_{v}=\{0\}$. So suppose $y(\lambda) \in \operatorname{ker} \mathcal{L}_{v}$. Then $0=\mathcal{L}_{v}(y(\lambda))=z_{1}(\lambda)$ by (5.5), which by (5.7) implies that $\widetilde{z}(\lambda)=0$, and hence that $z(\lambda)=0$. Therefore $0=\left(M^{T} \otimes I_{n}\right) z(\lambda)=y(\lambda)$ by (5.4), and so $\operatorname{ker} \mathcal{L}_{v}=\{0\}$.

Finally, we see why $\mathcal{L}_{v}$ induces a bijection on vector polynomials. First observe that the structure of the mapping $\mathcal{L}_{v}$ immediately implies that for any vector polynomial $y(\lambda) \in \mathcal{N}_{\ell}(L), \mathcal{L}_{v}(y(\lambda))$ will also be a vector polynomial. For the reverse direction, suppose that $\mathcal{L}_{v}(y(\lambda))=z_{1}(\lambda)$ is a vector polynomial. Then by (5.7) it is clear that $\widetilde{z}(\lambda)$ is also a vector polynomial, and hence so is $z(\lambda)$. Thus $y(\lambda)=\left(M^{T} \otimes I_{n}\right) z(\lambda)$ is a vector polynomial, and the proof is complete.

A counterpart of Lemma 5.5 for the right null vectors of linearizations in $\mathbb{L}_{2}(P)$ with full $Z$-rank can be proved in an analogous fashion. This is Lemma 5.6.

Lemma 5.6. Let $P(\lambda)$ be an $n \times n$ matrix polynomial of degree $k$, and suppose $L(\lambda) \in \mathbb{L}_{2}(P)$ has full $Z$-rank and left ansatz vector $w \neq 0$. Then the mapping

$$
\begin{aligned}
\mathcal{R}_{w}: \mathcal{N}_{r}(L) & \longrightarrow \mathcal{N}_{r}(P) \\
x(\lambda) & \longmapsto\left(w^{T} \otimes I_{n}\right) x(\lambda)
\end{aligned}
$$

is a linear isomorphism of $\mathbb{F}(\lambda)$-vector spaces. Furthermore, $\mathcal{R}_{w}$ induces a bijection between the vector polynomials in $\mathcal{N}_{r}(L)$ and the vector polynomials in $\mathcal{N}_{r}(P)$, i.e., $x(\lambda) \in \mathcal{N}_{r}(L)$ is a vector polynomial if and only if $\left(w^{T} \otimes I_{n}\right) x(\lambda) \in \mathcal{N}_{r}(P)$ is a vector polynomial.

Now we can state the main result concerning the recovery of left minimal indices and bases of a matrix polynomial $P(\lambda)$ from full $Z$-rank linearizations in $\mathbb{L}_{1}(P)$; this is Theorem 5.7. The analogous result for recovering right minimal indices and bases of $P(\lambda)$ from full $Z$-rank linearizations in $\mathbb{L}_{2}(P)$ is then Theorem 5.8. Since the arguments for both theorems are similar, we prove only Theorem 5.8.

TheOREM 5.7. Let $P(\lambda)$ be an $n \times n$ matrix polynomial of degree $k$, and let $L(\lambda) \in \mathbb{L}_{1}(P)$ have full $Z$-rank and nonzero right ansatz vector $v$. Then:

(a) The left minimal indices of $L(\lambda)$ and $P(\lambda)$ are the same.

(b) Every minimal basis of $\mathcal{N}_{\ell}(P)$ is of the form $\left\{\left(v^{T} \otimes I_{n}\right) y_{1}(\lambda), \ldots,\left(v^{T} \otimes\right.\right.$ $\left.\left.I_{n}\right) y_{p}(\lambda)\right\}$, where $\left\{y_{1}(\lambda), \ldots, y_{p}(\lambda)\right\}$ is a minimal basis of $\mathcal{N}_{\ell}(L)$.

THEOREM 5.8. Let $P(\lambda)$ be an $n \times n$ matrix polynomial of degree $k$, and let $L(\lambda) \in \mathbb{L}_{2}(P)$ have full $Z$-rank and nonzero left ansatz vector $w$. Then:

(a) The right minimal indices of $L(\lambda)$ and $P(\lambda)$ are the same.

(b) Every minimal basis of $\mathcal{N}_{r}(P)$ is of the form $\left\{\left(w^{T} \otimes I_{n}\right) x_{1}(\lambda), \ldots,\left(w^{T} \otimes\right.\right.$ $\left.\left.I_{n}\right) x_{p}(\lambda)\right\}$, where $\left\{x_{1}(\lambda), \ldots, x_{p}(\lambda)\right\}$ is a minimal basis of $\mathcal{N}_{r}(L)$.

Proof. We know from Lemma 5.6 that the mapping $\mathcal{R}_{w}$ defined in (5.8) is a linear isomorphism that induces a bijection between the vector polynomials in $\mathcal{N}_{r}(L)$ and those in $\mathcal{N}_{r}(P)$. Therefore $\mathcal{R}_{w}$ also induces a one-to-one correspondence between the polynomial bases of $\mathcal{N}_{r}(L)$ and those of $\mathcal{N}_{r}(P)$. If we can show that $\mathcal{R}_{w}$ preserves the degree of all vector polynomials in $\mathcal{N}_{r}(L)$, then it follows that this mapping establishes a bijection between minimal bases of $\mathcal{N}_{r}(L)$ and $\mathcal{N}_{r}(P)$, and the theorem will be proved. The following argument shows that $\mathcal{R}_{w}$ is indeed degree-preserving. 
Let $K \in \mathbb{F}^{k \times k}$ be a nonsingular matrix such that $w^{T} K=e_{1}^{T}$. Then by Lemma 5.6 every vector polynomial $z(\lambda) \in \mathcal{N}_{r}(P)$ can be written as

$$
z(\lambda)=\mathcal{R}_{w}(x(\lambda))=\left(w^{T} \otimes I_{n}\right)\left(K \otimes I_{n}\right)\left(K^{-1} \otimes I_{n}\right) x(\lambda),
$$

where $x(\lambda) \in \mathcal{N}_{r}(L)$ is also a vector polynomial. Hence

$$
z(\lambda)=\left(e_{1}^{T} \otimes I_{n}\right)\left(K^{-1} \otimes I_{n}\right) x(\lambda)=\left(e_{1}^{T} \otimes I_{n}\right) \widehat{x}(\lambda),
$$

where $\widehat{x}(\lambda):=\left(K^{-1} \otimes I_{n}\right) x(\lambda)$ is again a vector polynomial. Consideration of the leading coefficient vectors of $\widehat{x}(\lambda)$ and $x(\lambda)$ shows that $\operatorname{deg} \widehat{x}(\lambda)=\operatorname{deg} x(\lambda)$. Partitioning $\widehat{x}(\lambda)$ as $\left[\widehat{x}_{1}^{T}(\lambda), \ldots, \widehat{x}_{k}^{T}(\lambda)\right]^{T}$ with $\widehat{x}_{i}(\lambda) \in \mathbb{F}(\lambda)^{n}$, we see from (5.9) that $z(\lambda)=\widehat{x}_{1}(\lambda)$. Now the proof will be complete once we show that $\operatorname{deg} z(\lambda)=\operatorname{deg} \widehat{x}_{1}(\lambda)$ is the same as $\operatorname{deg} \widehat{x}(\lambda)=\operatorname{deg} x(\lambda)$, i.e., once we show that $\operatorname{deg} \widehat{x}_{1}(\lambda)$ and $\operatorname{deg} \widehat{x}(\lambda)$ are equal. Now clearly $\operatorname{deg} \widehat{x}_{1}(\lambda) \leq \operatorname{deg} \widehat{x}(\lambda)$, so all that remains is to establish that $\operatorname{deg} \widehat{x}_{1}(\lambda)<\operatorname{deg} \widehat{x}(\lambda)$ is impossible. For purposes of contradiction, then, let us assume that $\operatorname{deg} \widehat{x}_{1}(\lambda)<\operatorname{deg} \widehat{x}(\lambda)$ holds, and let $d:=\operatorname{deg} \widehat{x}(\lambda)$. Then we can write

$$
\widehat{x}(\lambda)=\left[\begin{array}{l}
0 \\
t
\end{array}\right] \lambda^{d}+(\text { lower order terms }),
$$

where $t \in \mathbb{F}^{(k-1) n}$ in the leading coefficient is a nonzero constant vector. Now since $x(\lambda) \in \mathcal{N}_{r}(L)$, we have $0=L(\lambda) x(\lambda)=L(\lambda)\left(K \otimes I_{n}\right) \widehat{x}(\lambda)$; using Theorem 4.6 on $L(\lambda)\left(K \otimes I_{n}\right)$ this can be written as

$$
\left(\lambda\left[\begin{array}{cc}
A_{k} & 0 \\
X_{21} & -Z
\end{array}\right]+\left[\begin{array}{cc}
Y_{11} & Z \\
A_{0} & 0
\end{array}\right]\right) \cdot \widehat{x}(\lambda) \equiv 0
$$

Extracting the coefficient of the highest degree (i.e. degree $d+1$ ) term from the left hand side of (5.10), we see that

$$
\left[\begin{array}{cc}
A_{k} & 0 \\
X_{21} & -Z
\end{array}\right]\left[\begin{array}{l}
0 \\
t
\end{array}\right]=\left[\begin{array}{c}
0 \\
-Z t
\end{array}\right]=0 .
$$

But $Z$ is nonsingular since $L(\lambda)$ has full $Z$-rank, so $t=0$; this contradiction to $t$ being nonzero then completes the proof.

The results in Theorems 5.2, 5.4, 5.7, and 5.8 allow us to recover the whole singular structure of a matrix polynomial $P(\lambda)$ from that of a full $Z$-rank linearization in either $\mathbb{L}_{1}(P)$ or $\mathbb{L}_{2}(P)$. But it turns out that full $Z$-rank is not a necessary condition for these relationships between minimal indices to hold. Example 2 exhibits a linearization in $\mathbb{L}_{1}(P)$ with deficient $Z$-rank having the same left and right minimal indices as a full $Z$-rank linearization from $\mathbb{L}_{1}(P)$ would have; the left minimal indices are the same as those of $P$, and the right minimal indices are shifted by $k-1$ from those of $P$. It is easy to check that $L(\lambda)$ in that example has one right minimal index $\widetilde{\varepsilon}_{1}=\varepsilon_{1}+1=1$ and one left minimal index $\widetilde{\eta}_{1}=\eta_{1}=0$. On the other hand, the fact that the left minimal index of $L(\lambda)$ in that example is equal to zero is not an accident, as the following elementary result shows.

Lemma 5.9. Suppose $L(\lambda) \in \mathbb{L}_{1}(P)$ has deficient $Z$-rank; i.e., the $Z$-rank is equal to $r$ and $r<(k-1) n$, so that $d:=(k-1) n-r$ is the Z-rank deficiency of $L(\lambda)$. Then $L(\lambda)$ has at least d left minimal indices equal to zero.

Proof. Since the matrix $Z \in \mathbb{F}^{(k-1) n \times(k-1) n}$ in (4.3) has rank $r$, there are $d$ linearly independent constant vectors $y_{1}, \ldots, y_{d}$ satisfying $y_{j}^{T} Z=0$ for $j=1, \ldots, d$. 
Set $\widetilde{y}_{j}=\left[\begin{array}{ll}0 & y_{j}^{T}\end{array}\right]^{T}$, where the first 0 block consists of $n$ zero entries, and define $z_{j}=\left(M \otimes I_{n}\right)^{T} \widetilde{y}_{j}$ for $j=1, \ldots, d$, where $M$ is the matrix in (4.3). Then $z_{1}, \ldots, z_{d}$ are linearly independent constant vectors satisfying $z_{j}^{T} L(\lambda) \equiv 0$ for $j=1, \ldots, d$. Thus $L(\lambda)$ has at least $d$ linearly independent left null vectors with degree zero, and hence at least $d$ left minimal indices equal to zero.

As a consequence of Lemma 5.9 , we see that a pencil $L(\lambda)$ in $\mathbb{L}_{1}(P)$ with $Z$-rank deficiency $d$ can have the same left minimal indices as $P(\lambda)$ only if $P(\lambda)$ itself has at least $d$ left minimal indices equal to zero.

As usual, there is a counterpart of Lemma 5.9 for pencils in $\mathbb{L}_{2}(P)$ and right minimal indices.

5.3. Summary of main results. For the convenience of the reader we gather together here the main results from Sections 5.1 and 5.2 on minimal index and minimal basis recovery, focusing on the generic case of "full $Z$-rank" linearizations in $\mathbb{L}_{1}(P)$ and $\mathbb{L}_{2}(P)$. Note that this includes the important special cases of the companion forms $C_{1}(\lambda) \in \mathbb{L}_{1}(P)$ and $C_{2}(\lambda) \in \mathbb{L}_{2}(P)$ described in (4.2). We restrict attention to full $Z$-rank linearizations in the interest of simplicity, so as to more clearly highlight the similarities and differences between linearizations in $\mathbb{L}_{1}(P)$ and those in $\mathbb{L}_{2}(P)$.

THEOREM 5.10 (Minimal indices and bases of full $Z$-rank linearizations). Let $P(\lambda)$ be an $n \times n$ matrix polynomial of degree $k$. Suppose $L_{1}(\lambda) \in \mathbb{L}_{1}(P)$ with right ansatz vector $v \neq 0$ and $L_{2}(\lambda) \in \mathbb{L}_{2}(P)$ with left ansatz vector $w \neq 0$ are linearizations of $P(\lambda)$ with full $Z$-rank. Then:

(a) The minimal indices of $P(\lambda), L_{1}(\lambda)$, and $L_{2}(\lambda)$ are related as follows.

\begin{tabular}{c|c|c} 
& Left minimal indices & Right minimal indices \\
\hline$P(\lambda)$ & $\eta_{1} \leq \eta_{2} \leq \cdots \leq \eta_{p}$ & $\varepsilon_{1} \leq \varepsilon_{2} \leq \cdots \leq \varepsilon_{p}$ \\
$L_{1}(\lambda) \in \mathbb{L}_{1}(P)$ & $\eta_{1} \leq \eta_{2} \leq \cdots \leq \eta_{p}$ & $\varepsilon_{1}+k-1 \leq \cdots \leq \varepsilon_{p}+k-1$ \\
$L_{2}(\lambda) \in \mathbb{L}_{2}(P)$ & $\eta_{1}+k-1 \leq \cdots \leq \eta_{p}+k-1$ & $\varepsilon_{1} \leq \varepsilon_{2} \leq \cdots \leq \varepsilon_{p}$
\end{tabular}

(b) The following table displays correspondences between minimal bases of $P(\lambda)$ and those of full $Z$-rank linearizations in $\mathbb{L}_{1}(P)$ with right ansatz vector $v$. (Note that the first companion form $C_{1}(\lambda)$ is one such linearization, with right ansatz vector $v=e_{1}$.)

\begin{tabular}{c|c|c} 
& Left minimal bases & Right minimal bases \\
\hline$P(\lambda)$ & $\left(v^{T} \otimes I_{n}\right) y_{1}(\lambda), \ldots,\left(v^{T} \otimes I_{n}\right) y_{p}(\lambda)$ & $x_{1}(\lambda), \ldots, x_{p}(\lambda)$ \\
$\uparrow$ & $\uparrow$ \\
$L_{1}(\lambda) \in \mathbb{L}_{1}(P)$ & $y_{1}(\lambda), \ldots, y_{p}(\lambda)$ & $\Lambda \otimes x_{1}(\lambda), \ldots, \Lambda \otimes x_{p}(\lambda)$
\end{tabular}

Each indicated correspondence describes a bijection between the set of all possible (left or right) minimal bases of the polynomial $P(\lambda)$ and the corresponding set of all possible (left or right) minimal bases of $L_{1}(\lambda) \in \mathbb{L}_{1}(P)$. The following table gives analogous correspondences for full $Z$-rank linearizations in $\mathbb{L}_{2}(P)$ with left ansatz vector $w$. (This includes the second companion form $C_{2}(\lambda)$, with $w=e_{1}$.) 


\begin{tabular}{c|c|c} 
& Left minimal bases & Right minimal bases \\
\hline$P(\lambda)$ & $y_{1}(\lambda), \ldots, y_{p}(\lambda)$ & $\left(w^{T} \otimes I_{n}\right) x_{1}(\lambda), \ldots,\left(w^{T} \otimes I_{n}\right) x_{p}(\lambda)$ \\
& $\uparrow$ & $\uparrow$ \\
$L_{2}(\lambda) \in \mathbb{L}_{2}(P)$ & $\Lambda \otimes y_{1}(\lambda), \ldots, \Lambda \otimes y_{p}(\lambda)$ & $x_{1}(\lambda), \ldots, x_{p}(\lambda)$
\end{tabular}

Proof. The results follow from Theorems 5.2, 5.4, 5.7, and 5.8.

A striking consequence of Theorem 5.10(a) concerns the relationship between the companion forms $C_{1}(\lambda)$ and $C_{2}(\lambda)$ of a singular polynomial. It is well known that any two strong linearizations of a regular polynomial $P(\lambda)$ are strictly equivalent [16, 23], i.e., equivalent in the sense of Definition 2.2 but with nonsingular constant matrices $E$ and $F$. In particular, the first and second companion forms of a regular $P(\lambda)$ are always strictly equivalent. The situation for singular $P(\lambda)$, though, is completely different.

Corollary 5.11. For a singular $n \times n$ matrix polynomial $P(\lambda)$ of degree $k \geq 2$, the first and second companion forms $C_{1}(\lambda)$ and $C_{2}(\lambda)$ are never strictly equivalent.

Proof. It is easy to see that strictly equivalent polynomials must have exactly the same minimal indices. But from Theorem 5.10(a) it is clear that for any singular polynomial $P$ of degree $k \geq 2$, any full $Z$-rank linearization from $\mathbb{L}_{1}(P)$ will have different minimal indices from those of any full $Z$-rank linearization from $\mathbb{L}_{2}(P)$. Since $C_{1}(\lambda) \in \mathbb{L}_{1}(P)$ and $C_{2}(\lambda) \in \mathbb{L}_{2}(P)$ both have full $Z$-rank, they have different minimal indices, and therefore cannot be strictly equivalent.

6. Linearizations in $\mathbb{D L}(P)$ for singular $P$ ? . For a matrix polynomial $P(\lambda)$ of degree $k$, it was proved in $[20,25]$ that the dimension of $\mathbb{D L}(P):=\mathbb{L}_{1}(P) \cap \mathbb{L}_{2}(P)$ is $k$, and furthermore that if $P(\lambda)$ is regular then almost all pencils in $\mathbb{D L}(P)$ are linearizations of $P(\lambda)$. This is in stark contrast with the situation when $P(\lambda)$ is singular, as we show in Theorem 6.1.

TheOREM 6.1. If $P(\lambda)$ is an $n \times n$ singular matrix polynomial of degree $k \geq 2$, then none of the pencils in $\mathbb{D L}(P)$ is a linearization of $P(\lambda)$.

Proof. Suppose $L(\lambda) \in \mathbb{D L}(P)$ is a linearization of $P(\lambda)$. The only pencil in $\mathbb{D L}(P)$ with either left or right ansatz vector being zero is the zero pencil [20, Thm. 3.4], which by Definition 2.2 can clearly never be a linearization for $P(\lambda)$. Thus both the left and right ansatz vectors of $L(\lambda)$ must be nonzero. Since $L(\lambda) \in \mathbb{L}_{2}(P)$, Theorem 5.4 implies that the left minimal indices of $L(\lambda)$ are $0<(k-1)+\eta_{1} \leq \cdots \leq(k-1)+\eta_{p}$, where $\eta_{1} \leq \cdots \leq \eta_{p}$ are the left minimal indices of $P(\lambda)$. But $L(\lambda)$ is also in $\mathbb{L}_{1}(P)$, which leads to a contradiction with Theorem 5.7 if $L(\lambda)$ has full $Z$-rank, or with Lemma 5.9 if $L(\lambda)$ does not have full $Z$-rank.

7. Linearizations defined via other polynomial bases. In some contexts it may be useful to consider polynomial bases other than the standard monomial basis $\left\{1, \lambda, \ldots, \lambda^{k-1}\right\}$ (see, for instance, [2] and the references therein). In this section, we begin by generalizing the vector space $\mathbb{L}_{1}(P)$ as in [24, Section 4.2], modifying the defining right ansatz condition in (4.1) by using an arbitrary scalar polynomial basis to replace the standard monomial basis in the vector $\Lambda=\left[\lambda^{k-1}, \lambda^{k-2}, \ldots, \lambda, 1\right]^{T}$. Then we show that these new pencils are strictly equivalent to the pencils in $\mathbb{L}_{1}(P)$, and so most of them are strong linearizations of $P(\lambda)$ that can be used to recover the minimal indices and bases of $P(\lambda)$. This new class of pencils includes ones that are associated with matrix polynomials expressed in non-monomial polynomial bases. In particular, it follows from our results that the pencils introduced in [2, Sections 2 
and 3] using degree-graded polynomial bases are strong linearizations of $P(\lambda)$ even for singular $P(\lambda)$; note that in [2] only the regular case was considered. We focus here only on generalizing the pencils in $\mathbb{L}_{1}(P)$, but similar results can be obtained for an analogous generalization of the pencils in $\mathbb{L}_{2}(P)$.

Let $\phi_{0}(\lambda), \phi_{1}(\lambda), \ldots, \phi_{k-1}(\lambda)$ be any basis for the space of all scalar polynomials of degree less than $k$, and set $\Lambda_{\phi}:=\left[\begin{array}{llll}\phi_{0}(\lambda) & \phi_{1}(\lambda) & \ldots & \phi_{k-1}(\lambda)\end{array}\right]^{T}$. Let $\Phi$ be the unique (nonsingular) constant matrix such that $\Lambda_{\phi}=\Phi \Lambda=\Phi\left[\lambda^{k-1}, \ldots, \lambda, 1\right]^{T}$. Then the following theorem provides the foundation for the main results in this section. It was stated and proved in [24], but we include it here for completeness.

Theorem 7.1. [24, Prop. 4.9] Let $P(\lambda)$ be an $n \times n$ matrix polynomial of degree $k$ and let $L(\lambda)=\lambda X+Y$ be any $k n \times k n$ pencil such that $L(\lambda)$ satisfies the modified right ansatz

$$
L(\lambda) \cdot\left(\Lambda_{\phi} \otimes I_{n}\right)=v \otimes P(\lambda)
$$

for some nonzero $v \in \mathbb{F}^{k}$. Let $M$ be any nonsingular matrix such that $M v=e_{1}$. If the lower right $(k-1) n \times(k-1) n$ principal submatrix of $\widetilde{X}$ in

$$
\widetilde{L}(\lambda)=\lambda \widetilde{X}+\widetilde{Y}:=\left(M \otimes I_{n}\right) \cdot L(\lambda) \cdot\left(\Phi \otimes I_{n}\right)
$$

is nonsingular, then $L(\lambda)$ is a strong linearization for $P(\lambda)$.

Observe that in Theorem 7.1 the pencil $\widetilde{L}(\lambda)$ is in $\mathbb{L}_{1}(P)$, with right ansatz vector $e_{1}$. Thus we see that any pencil $L(\lambda)$ satisfying the modified right ansatz (7.1) will be strictly equivalent to a pencil in $\mathbb{L}_{1}(P)$.

Now we can state the first of the main results of this section, a generalization of Theorem 5.2 for linearizations satisfying the modified right ansatz (7.1).

TheOREM 7.2. Let $P(\lambda)$ be an $n \times n$ matrix polynomial of degree $k$, and let $L(\lambda)$ be a linearization of $P(\lambda)$ satisfying the modified right ansatz (7.1) with $v \neq 0$.

(a) If $\varepsilon_{1} \leq \cdots \leq \varepsilon_{p}$ are the right minimal indices of $P(\lambda)$, then

$$
(k-1)+\varepsilon_{1} \leq(k-1)+\varepsilon_{2} \leq \cdots \leq(k-1)+\varepsilon_{p} .
$$

are the right minimal indices of $L(\lambda)$.

(b) Every minimal basis of $\mathcal{N}_{r}(L)$ is of the form $\left\{\Lambda_{\phi} \otimes x_{1}(\lambda), \ldots, \Lambda_{\phi} \otimes x_{p}(\lambda)\right\}$, where $\left\{x_{1}(\lambda), \ldots, x_{p}(\lambda)\right\}$ is a minimal basis of $\mathcal{N}_{r}(P)$.

Proof. As noted right after Theorem 7.1, $L(\lambda)$ is strictly equivalent to a linearization in $\mathbb{L}_{1}(P)$, and so must have the same right minimal indices as any linearization in $\mathbb{L}_{1}(P)$. Part (a) then follows from Theorem 5.2(a).

Using Theorem 5.2 again on the pencil $\widetilde{L}(\lambda)$ in $(7.2)$, we see that every minimal basis of $\mathcal{N}_{r}(\widetilde{L})$ is of the form $\left\{\Lambda \otimes x_{1}(\lambda), \ldots, \Lambda \otimes x_{p}(\lambda)\right\}$ for some minimal basis $\left\{x_{1}(\lambda), \ldots, x_{p}(\lambda)\right\}$ of $\mathcal{N}_{r}(P)$. Now $(7.2)$ implies that multiplying any basis of $\mathcal{N}_{r}(\widetilde{L})$ on the left by the nonsingular matrix $\Phi \otimes I_{n}$ will produce a basis of $\mathcal{N}_{r}(L)$, and that all bases of $\mathcal{N}_{r}(L)$ can be obtained in this way. Since $\Phi \otimes I_{n}$ is nonsingular and constant, left multiplication does not change the degree of any vector polynomial, so minimal bases of $\mathcal{N}_{r}(\widetilde{L})$ turn into minimal bases of $\mathcal{N}_{r}(L)$. Part (b) then follows, because $\Lambda_{\phi}=\Phi \Lambda$. $\square$

Note that according to Theorem 7.2, the recovery of a minimal basis of $\mathcal{N}_{r}(P)$ from a minimal basis of $\mathcal{N}_{r}(L)$ requires division of $n p$ scalar polynomials by one of the basis polynomials $\phi_{j}(\lambda)$. This is not a trivial task if there are no monomials in the basis $\left\{\phi_{0}(\lambda), \phi_{1}(\lambda), \ldots, \phi_{k-1}(\lambda)\right\}$.

There is also an analog of Theorem 5.7 for pencils satisfying the modified right ansatz (7.1). This is Theorem 7.3. 
THEOREM 7.3. Let $P(\lambda)$ be an $n \times n$ matrix polynomial of degree $k$, and let $L(\lambda)$ be a matrix pencil satisfying all the hypotheses of Theorem 7.1. Then:

(a) The left minimal indices of $L(\lambda)$ and $P(\lambda)$ are the same.

(b) Every minimal basis of $\mathcal{N}_{\ell}(P)$ is of the form $\left\{\left(v^{T} \otimes I_{n}\right) z_{1}(\lambda), \ldots,\left(v^{T} \otimes\right.\right.$ $\left.\left.I_{n}\right) z_{p}(\lambda)\right\}$, where $\left\{z_{1}(\lambda), \ldots, z_{p}(\lambda)\right\}$ is a minimal basis of $\mathcal{N}_{\ell}(L)$.

Proof. As in Theorem 7.2, part (a) is a consequence of Theorem 5.7(a) and the invariance of minimal indices under strict equivalence, since $\widetilde{L}(\lambda)$ in $(7.2)$ is in $\mathbb{L}_{1}(P)$ with nonzero right ansatz vector and full $Z$-rank. To prove part (b), first observe from (7.2) that $\widetilde{y}(\lambda)$ is a left null vector of $\widetilde{L}(\lambda)$ if and only if $z(\lambda)=\left(M \otimes I_{n}\right)^{T} \widetilde{y}(\lambda)$ is a left null vector of $L(\lambda)$. Applying Theorem 5.7 to $\widetilde{L}(\lambda)$, we see that every minimal basis of $\mathcal{N}_{\ell}(P)$ is of the form $\left\{\left(e_{1}^{T} \otimes I_{n}\right) \widetilde{y}_{1}(\lambda), \ldots,\left(e_{1}^{T} \otimes I_{n}\right) \widetilde{y}_{p}(\lambda)\right\}$, where $\left\{\widetilde{y}_{1}(\lambda), \ldots, \widetilde{y}_{p}(\lambda)\right\}$ is a minimal basis of $\mathcal{N}_{\ell}(\widetilde{L})$, equivalently, where $\left\{\left(M \otimes I_{n}\right)^{T} \widetilde{y}_{1}(\lambda), \ldots,\left(M \otimes I_{n}\right)^{T} \widetilde{y}_{p}(\lambda)\right\}$ is a minimal basis of $\mathcal{N}_{\ell}(L)$. Part (b) now follows by taking $z_{i}(\lambda)$ to be $\left(M \otimes I_{n}\right)^{T} \widetilde{y}_{i}(\lambda)$ for $i=1, \ldots, p$, and recalling that $e_{1}^{T} M^{-T}=v^{T}$.

We finish by applying the results in this section to show that the pencils in [2, Sections 2 and 3] defined in terms of degree-graded polynomial bases are strong linearizations of $P(\lambda)$, and that they can be used to recover the minimal indices and bases of $P(\lambda)$ through Theorems 7.2 and 7.3. The phrase "degree-graded" here refers to polynomial bases $\phi_{0}(\lambda), \phi_{1}(\lambda), \ldots, \phi_{k-1}(\lambda)$ with the property that $\operatorname{deg} \phi_{j}(\lambda)=j$ for $j=0, \ldots,(k-1)$. The pencils in [2, Section 2] satisfy a generalized left ansatz version of (7.1). However, the block transposes of the pencils in [2, Section 2] satisfy (7.1) with $v=c e_{k}$, where $c$ is a nonzero constant and $e_{k}$ is the $k$ th column of $I_{k}$. So for these pencils the matrix $M$ in (7.2) can be taken as $M=c^{-1} R$, where $R$ is the matrix obtained by reversing the order of the rows of $I_{k}$, and the change of basis matrix $\Phi$ is nonsingular and lower anti-triangular (because of the degree-grading). In addition, if $L(\lambda)=\lambda X+Y$ is the block transpose of a pencil in [2, Section 2] then $X=\operatorname{diag}\left(I_{n(k-1)}, b A_{k}\right)$, with $b$ a nonzero constant. As a consequence of these arguments, the matrix $\tilde{X}$ in (7.2) satisfies

$$
\begin{aligned}
\widetilde{X} & =c^{-1}\left(R \otimes I_{n}\right) \cdot \operatorname{diag}\left(I_{n(k-1)}, b A_{k}\right) \cdot\left(\Phi \otimes I_{n}\right) \\
& =c^{-1} \operatorname{diag}\left(b A_{k}, I_{n(k-1)}\right) \cdot\left(R \otimes I_{n}\right) \cdot\left(\Phi \otimes I_{n}\right) \\
& =c^{-1} \operatorname{diag}\left(b A_{k}, I_{n(k-1)}\right) \cdot\left(R \Phi \otimes I_{n}\right),
\end{aligned}
$$

where $R \Phi$ is nonsingular upper triangular. Thus the lower right $(k-1) n \times(k-1) n$ principal submatrix of $\tilde{X}$ is also nonsingular, so that Theorems 7.1, 7.2, and 7.3 hold for the block transposes of the pencils in [2, Section 2 and 3].

Note that results similar to Theorems $7.1,7.2$, and 7.3 may also be developed for pencils satisfying a modified left ansatz analogous to (7.1), showing how such pencils are closely connected to pencils in $\mathbb{L}_{2}(P)$, and how recovery from them of minimal indices and bases for $P$ may be achieved. Such results can then be applied directly (without any block transposing) to the pencils in [2, Section 2 and 3].

8. Conclusions and future work. We have proved that almost all of the pencils in the vector spaces $\mathbb{L}_{1}(P)$ and $\mathbb{L}_{2}(P)$ introduced in [25], and all of the pencils introduced in [2, Sections 2 and 3], are strong linearizations for any $n \times n$ singular matrix polynomial $P(\lambda)$, but that none of the pencils in $\mathbb{D L}(P)$ is ever a linearization for such a $P(\lambda)$. In addition, we have shown how to recover the minimal indices and bases of $P(\lambda)$ from the minimal indices and bases of linearizations in $\mathbb{L}_{1}(P)$, in $\mathbb{L}_{2}(P)$, and in [2, Sections 2 and 3]. This suggests the possibility of computing these quantities from the GUPTRI form of a linearization, as was done in [6] for the companion 
form of full-rank rectangular matrix polynomials that have minimal indices only on one side.

The results in this paper on linearizations from $\mathbb{L}_{1}(P)$ and $\mathbb{L}_{2}(P)$ for singular polynomials have been extended to include the companion-like pencils that were introduced in [4] as linearizations for regular polynomials. These extended results will be presented in the forthcoming paper [12].

\section{REFERENCES}

[1] A. Amiraslani, D. A. Aruliah and R. M. Corless, Block LU factors of generalized companion matrix pencils, Theor. Comp. Science, 381 (2007), pp. 134-147.

[2] A. Amiraslani, R. M. Corless and P. Lancaster, Linearization of matrix polynomials expressed in polynomial bases, IMA J. Num. Anal., 29 (2009), pp. 141-157.

[3] E. N. Antoniou, A. I. G. Vardulakis, and S. Vologiannidis, Numerical computation of minimal polynomial bases: A generalized resultant approach, Linear Algebra Appl., 405 (2005), pp. 264-278.

[4] E. N. Antoniou And S. Vologiannidis, A new family of companion forms of polynomial matrices, Electron. J. Linear Algebra, 11 (2004), pp. 78-87.

[5] E. N. Antoniou And S. Vologiannidis, Linearizations of polynomial matrices with symmetries and their applications, Electron. J. Linear Algebra, 15 (2006), pp. 107-114.

[6] T. G. Beelen and G. W. Veltkamp, Numerical computation of a coprime factorization of a transfer function matrix, Systems Control Lett., 9 (1987), pp. 281-288.

[7] R. Byers, V. Mehrmann, and H. Xu, Trimmed linearizations for structured matrix polynomials, Linear Algebra Appl., 429 (2008), pp. 2373-2400.

[8] J. W. Demmel And B. KÅGSTRÖM, The generalized Schur decomposition of an arbitrary pencil $A-\lambda B$ : Robust software with error bounds and applications. Part I: Theory and Algorithms, ACM T. Math. Software, 19 (1993), pp. 160-174.

[9] J. W. Demmel And B. KÅGström, The generalized Schur decomposition of an arbitrary pencil $A-\lambda B$ : Robust software with error bounds and applications. Part II: Software and Applications, ACM T. Math. Software, 19 (1993), pp. 175-201.

[10] F. De Terán And F. M. Dopico, Low rank perturbation of Kronecker structures without full rank, SIAM J. Matrix Anal. Appl., 29 (2007), pp. 496-529.

[11] F. De Terán And F. M. Dopico, Sharp lower bounds for the dimension of linearizations of matrix polynomials, Electron. J. Linear Algebra, 17 (2008), pp. 518-531.

[12] F. De Terán, F. M. Dopico, And D. S. Mackey, Fiedler companion linearizations and the recovery of minimal indices, in preparation.

[13] A. Edelman, E. Elmroth, And B. KÅgström, A geometric approach to perturbation theory of matrices and matrix pencils. Part II. A stratification-enhanced staircase algorithm, SIAM J. Matrix Anal. Appl., 20 (1999), pp. 667-699

[14] G. D. Forney, Minimal bases of rational vector spaces, with applications to multivariable linear systems, SIAM J. Control, 13 (1975), pp. 493-520.

[15] F. R. Gantmacher, The Theory of Matrices, AMS Chelsea, Providence, RI, 1998.

[16] I. Gohberg, M. A. KaAshoek, And P. Lancaster, General theory of regular matrix polynomials and band Toeplitz operators, Integr. Eq. Operator Theory, 11 (1988), pp. 776-882.

[17] I. Gohberg, P. Lancaster, And L. Rodman, Matrix Polynomials, Academic Press, New York, 1982.

[18] G. E. Hayton, A. C. Pugh, and P. Fretwell, Infinite elementary divisors of a matrix polynomial and implications, Int. J. Control, 47 (1988), pp. 53-64.

[19] N. J. Higham, D. S. Mackey, And F. Tisseur, The conditioning of linearizations of matrix polynomials, SIAM J. Matrix Anal. Appl., 28 (2006), pp. 1005-1028.

[20] N. J. Higham, D. S. Mackey, N. Mackey, and F. Tisseur, Symmetric linearizations for matrix polynomials, SIAM J. Matrix Anal. Appl., 29 (2006), pp. 143-159.

[21] N. J. Higham, R-C. Li, AND F. Tisseur, Backward error of polynomial eigenproblems solved by linearization, SIAM J. Matrix Anal. Appl., 29, no. 4 (2007), pp. 1218-1241.

[22] T. Kallath, Linear Systems, Prentice Hall, Englewood Cliffs, NJ, 1980.

[23] P. Lancaster and P. Psarrakos, A note on weak and strong linearizations of regular matrix polynomials. Available as MIMS-EPrint 2006.72, Manchester Institute for Mathematical Sciences.

[24] D. S. Mackey, N. Mackey, C. Mehl, and V. Mehrmann, Vector spaces of linearizations for matrix polynomials, preprint, April 2005. Available at 
http://www.matheon.de/research/list_preprints.asp

[25] D. S. Mackey, N. Mackey, C. Mehl, and V. Mehrmann, Vector spaces of linearizations for matrix polynomials, SIAM J. Matrix Anal. Appl., 28 (2006), pp. 971-1004.

[26] D. S. Mackey, N. Mackey, C. Mehl, and V. Mehrmann, Structured polynomial eigenvalue problems: Good vibrations from good linearizations, SIAM J. Matrix Anal. Appl., 28 (2006), pp. 1029-1051.

[27] V. Mehrmann and D. Watkins, Polynomial eigenvalue problems with Hamiltonian structure, Electr. Trans. Num. Anal., 13 (2002), pp. 106-118.

[28] P. VAN Dooren, The computation of Kronecker's canonical form of a singular pencil, Linear Algebra Appl., 27 (1979), pp. 103-140.

[29] P. VAn Dooren, Reducing subspaces: Definitions, properties, and algorithms. In Matrix Pencils, Lecture Notes in Mathematics, Vol. 973, B. Kågström and A. Ruhe, Eds., SpringerVerlag, Berlin, 1983, pp. 58-73.

[30] P. Van Dooren and P. Dewilde, The Eigenstructure of an Arbitrary Polynomial Matrix: Computational Aspects, Linear Algebra Appl., 50 (1983), pp. 545-579.

[31] J. C. ZÚÑIgA AND D. HenRion, A Toeplitz algorithm for polynomial J-spectral factorization, Automatica 42 (2006), pp. 1085-1093. 